\title{
Isoprenylcysteine carboxylmethyltransferase deficiency exacerbates KRAS-driven pancreatic neoplasia via Notch suppression
}

\author{
Helen Court, 1,2,3,4 Marc Amoyel, ${ }^{3}$ Michael Hackman, ${ }^{5}$ Kyoung Eun Lee, ${ }^{3}$ Ruliang Xu, ${ }^{6}$ \\ George Miller, ${ }^{5}$ Dafna Bar-Sagi, ${ }^{3,4}$ Erika A. Bach, ${ }^{3}$ Martin O. Bergö,7 and Mark R. Philips',2,3,4 \\ ${ }^{1}$ Department of Medicine, ${ }^{2}$ Department of Cell Biology, ${ }^{3}$ Department of Biochemistry and Molecular Pharmacology, ${ }^{4}$ Cancer Institute, \\ ${ }^{5}$ Department of Surgery, and ${ }^{6}$ Department of Pathology, NYU School of Medicine, New York, New York, USA. \\ ${ }^{7}$ Institute of Medicine, Sahlgrenska University Hospital, Gothenburg, Sweden.
}

\begin{abstract}
RAS is the most frequently mutated oncogene in human cancers. Despite decades of effort, anti-RAS therapies have remained elusive. Isoprenylcysteine carboxylmethyltransferase (ICMT) methylates RAS and other CaaX-containing proteins, but its potential as a target for cancer therapy has not been fully evaluated. We crossed a Pdx1-Cre;LSL-Kras ${ }^{G 12 D}$ mouse, which is a model of pancreatic ductal adenocarcinoma (PDA), with a mouse harboring a floxed allele of Icmt. Surprisingly, we found that ICMT deficiency dramatically accelerated the development and progression of neoplasia. ICMT-deficient pancreatic ductal epithelial cells had a slight growth advantage and were resistant to premature senescence by a mechanism that involved suppression of cyclin-dependent kinase inhibitor $2 \mathrm{~A}$ ( $\left.\mathrm{p} 16^{\mathrm{INK} 4 \mathrm{~A}}\right)$ expression. ICMT deficiency precisely phenocopied Notch1 deficiency in the $P d x 1$-Cre; $L S L-K r a s^{G 12 D}$ model by exacerbating pancreatic intraepithelial neoplasias, promoting facial papillomas, and derepressing Wnt signaling. Silencing ICMT in human osteosarcoma cells decreased Notch1 signaling in response to stimulation with cell-surface ligands. Additionally, targeted silencing of Ste14, the Drosophila homolog of Icmt, resulted in defects in wing development, consistent with Notch loss of function. Our data suggest that ICMT behaves like a tumor suppressor in PDA because it is required for Notch1 signaling.
\end{abstract}

\section{Introduction}

RAS is the most frequently mutated oncogene in human cancer (1). This has made RAS the target of drug discovery efforts for more than three decades, but effective anti-RAS therapies have remained elusive (2). RAS is a prototypical small GTPase that functions as a binary molecular switch to regulate a number of signaling pathways including those that control growth and differentiation. RAS is inactive when GDP bound and is active when it binds GTP. The GDP/GTP cycle is controlled by guanine nucleotide exchange factors (GEFs) that activate RAS and by GTPase-activating proteins (GAPs) that dramatically accelerate the rate of GTP hydrolysis catalyzed by RAS, thereby limiting RAS activity (3). The oncogenic mutations found in human cancer are one of several single nucleotide changes in codons 12,13 , or 61 that render RAS insensitive to GAPs and reduce the intrinsic rate of GTP hydrolysis, thus allowing the accumulation of GTP-bound, activated RAS $(1,4)$. Attempts to devise therapeutic agents that reverse the accumulation of GTP-bound RAS have failed. Accordingly, investigators have taken an alternate approach driven by another hallmark of RAS biology: its regulation of signaling pathways only when associated with cellular membranes (5).

RAS is a peripheral membrane protein that gains affinity for membranes as a consequence of a series of posttranslational modifications of a C-terminal CaaX motif (6). The CaaX sequence is first modified by farnesyltransferase (FTase), which adds a farnesyl lipid to the cysteine. Next, RAS-converting enzyme 1 (RCE1) removes the aaX amino acids. Finally, isoprenylcysteine carboxylmethyltransferase (ICMT) methyl esterifies the $\alpha$-carboxyl group of the newly

Conflict of interest: The authors have declared that no conflict of interest exists. Citation for this article: J Clin Invest. 2013;123(11):4681-4694. doi:10.1172/JCI65764. formed C-terminal farnesylcysteine. The end result of the three modifications is to render the $\mathrm{C}$ terminus of RAS hydrophobic with affinity for phospholipid bilayers (6). The three CaaX-modifying enzymes have long been considered targets for anti-RAS drug discovery because inhibiting one or more of the steps of this pathway should render RAS more cytosolic and thereby reduce signaling to downstream effectors. Indeed, FTase inhibitors (FTIs) were developed two decades ago (7). However, despite their favorable attributes as drugs and their success in blocking FTase activity in vivo, they proved ineffective as anticancer drugs because oncogenic RAS could remain active by modification with another prenyltransferase (8). This result focused attention on RCE1 and ICMT as alternative drug targets.

To validate ICMT as a target for anticancer drug discovery, we engineered mice with a conditional allele for the Icmt gene Icm $t^{f l x}$, in which exon 1 of Icmt is flanked by LoxP sites (9). Of the three RAS isoforms expressed in mammalian tissues, KRAS was selected for analysis because the vast majority of human cancers driven by oncogenic RAS are associated with mutations of the KRAS gene (1). When mouse embryonic fibroblasts (MEFs) from Icmt $t^{f x / f l x}$ animals were transfected with oncogenic Kras ${ }^{G 12 V}$, they became transformed. Importantly, when Icmt was ablated in the transformed cells by infection with an adenovirus directing the expression of Cre recombinase, the transformed phenotype was reverted and could be restored by reexpression of ICMT (9). This result was concordant with the effects of Icmt ablation on two in vivo models of KRAS-driven neoplasia, both of which used a latent, Cre-inducible oncogenic $L S L-K_{r a s}{ }^{G 12 D}$ allele (10). ICMT deficiency ameliorated the myeloproliferative disease observed in Mx1-Cre;LSL-Kras ${ }^{G 12 D}$ mice and prolonged survival in $L S L-K r a s^{G 12 D}$ mice bred with animals containing a lysozyme $M$-Cre allele that expresses Cre in type 2 pneumocytes and therefore produces lung tumors (10). 
These studies support the idea that ICMT is a good target for antiKRAS drug discovery. However, neither the myeloproliferative disease observed in Mx1-Cre;LSL-Kras ${ }^{G 12 D}$ mice, nor the lung tumors seen in M-Cre;LSL-Kras ${ }^{G 12 D}$ animals are genetically or histologically faithful to the analogous diseases seen in humans. In contrast, the neoplastic transformation and disease progression observed in the pancreas of $P d x 1$-Cre;LSL-Kras ${ }^{G 12 D}$ mice faithfully mimics the progression of human pancreatic ductal adenocarcinoma (PDA) through a series of precursor lesions known as pancreatic intraepithelial neoplasias (PanINs) $(11,12)$. Moreover, not only is PDA among the human cancers most frequently associated with oncogenic mutations in the KRAS gene (13), but activating mutations in the KRAS proto-oncogene have been found in patients with early PanIN lesions (14), suggesting that KRAS mutations may be an initiating genetic event for PDA. We therefore sought to further investigate the role of ICMT in KRAS-driven cancer using the Pdx1-Cre; LSL-Kras ${ }^{G 12 D}$ model of PDA. To accomplish this, we compared Icmt $t^{f x /+} ; P d x 1$-Cre;LSL-Kras ${ }^{G 12 D}$ with Icm $t^{f l x} / f x ; P d x 1$-Cre; $L S L-K r a s^{G 12 D}$ mice, expecting to see an amelioration of disease in the setting of ICMT deficiency. We were instead surprised to observe a marked acceleration of PanIN progression to PDA in ICMT-deficient pancreata. Despite partial subcellular mislocalization in pancreatic tissue, oncogenic KRAS signaling was preserved in the setting of ICMT deficiency. Notch1 is a known tumor suppressor in the Pdx1-Cre;LSL-Kras ${ }^{G 12 D}$ model of PDA $(15,16)$, and we found that ICMT deficiency phenocopied Notch1 deficiency and that efficient Notch signaling in both human tumor cells and Drosophila required ICMT. Thus, in the Pdx1-Cre;LSL-Kras ${ }^{G 12 D}$ model of pancreatic neoplasia, ICMT behaves like a tumor suppressor because it is required for Notch1 signaling.

\section{Results}

ICMT is not required for normal pancreatic development. In addition to the four isoforms of RAS, ICMT has as many as 300 potential substrates including a broad range of signaling molecules that regulate, among other processes, cell growth, the actin cytoskeleton, vesicular transport, and nuclear architecture (17-19). We therefore sought to determine whether pancreata can develop normally in mice genetically engineered to lose ICMT expression in the pancreas. To answer this question, we used mice transgenic for a $P d x 1$-Cre allele that drives expression of Cre recombinase under the control of the $P d x 1$ promoter (11). In these animals, Cre recombinase is expressed, beginning at E8.5, in pancreatic progenitor cells that give rise to adult acinar, ductal, and islet cells. We crossed the Pdx1-Cre mice with $I c m t^{f x / f l x}$ mice that are homozygous for an allele of Icmt in which the first exon is flanked by LoxP sites (floxed) (9). Cre-mediated deletion of the first exon of Icmt in mouse fibroblasts has been shown to result in a complete loss of the enzymatic activity and in an accumulation of unmethylated ICMT protein substrates (9). We bred mice with the genotypes Icmt $t^{f x /+} ; P d x 1$-Cre and Icmt $t^{f x / f l x} ; P d x 1$-Cre, in which either one or both Icmt genes are inactivated in the pancreas. Mice of both genotypes were born at the expected Mendelian ratios and gained weight normally. No evidence of pancreatic insufficiency or diabetes was observed (Supplemental Figure 1A). A cohort of mice $(n=4)$ was followed for 1 year and remained healthy. These mice were sacrificed and subjected to necropsy. The ICMT-deficient pancreata of the 1-year-old mice appeared histologically normal (Supplemental Figure 1B).

The $P d x 1$-Cre transgene has been reported to be expressed in a mosaic pattern in the pancreas $(11,20)$. We were therefore concerned that the normal pancreata in Icmt $t^{f l x} / f x ; P d x 1$-Cre mice might result from progenitor cells that escaped ablation of Icmt. In order to determine whether Cre recombinase is expressed efficiently in $P d x 1$-Cre transgenic pancreata, regardless of Icmt status, we crossed $I c m t^{f x /+} ; P d x 1$-Cre and Icmt ${ }^{f l x / f l x} ; P d x 1$-Cre mice with Rosa26-LSL-LacZ (R26R) reporter mice (21) and analyzed sections of pancreata for $\beta$-gal expression using X-gal staining. Whereas pancreatic tissue stained blue, extrapancreatic structures such as blood vessels and lymph nodes were negative for $\beta$-gal (Supplemental Figure 1C). We observed uniform expression of $\beta$-gal in the pancreata of $I c m t^{f l x /+}$; Pdx1-Cre;Rosa26-LSL-LacZ mice (Supplemental Figure 1C), indicating that $P d x 1$-Cre expression occurred throughout the pancreas and that heterozygosity at the Icmt locus did not affect pancreatic development. In contrast, whereas the majority of acinar, ductal, and islet cells in the pancreata of Icmt $t^{f x / f l x} ; P d x 1$-Cre; Rosa26-LSL-LacZ animals stained positively for $\beta$-gal, a minor population of pancreatic cells did not, confirming a mosaic expression of Cre and suggesting a subtle growth disadvantage for ICMT-deficient cells (Supplemental Figure 1C). Importantly, despite the mosaicism, in all animals examined, there was a substantial contribution of Cre-expressing ( $\beta$-gal-positive) cells to all major pancreatic structures including ducts, acini, and islets, indicating that ICMT-deficient cells were able to contribute to normal pancreatic development.

To confirm that recombination of the Icmt flx allele resulted in loss of enzymatic activity, we removed portions of pancreata from 2-month-old mice of the genotypes Icm t $t^{f l / f l x} ; P d x 1_{-}$-Cre and Icm $t^{f l x /+}$; Pdx1-Cre, homogenized the tissue, isolated crude membrane fractions, and assayed the membranes for ICMT activity (22). Whereas robust activity was detected in the membrane preparations from $I c m t^{f l x /+} ; P d x 1$-Cre mice, membranes from Icmt $t^{f l x / f l x} ; P d x 1$-Cre mice possessed very low activity (Supplemental Figure 1D). These results demonstrate that ICMT can be ablated in the embryonic pancreas without developmental consequences.

ICMT deficiency exacerbates PanIN development in a mouse model of PDA. To determine the role of ICMT in KRAS-driven PDA, we chose a mouse model that faithfully recapitulates the progression of human disease by inducing PanINs (11). LSL-Kras G12D mice have a Kras gene with a G12D-activating mutation knocked into the Kras locus. Importantly, the oncogene is preceded by a stop transcription (S) site flanked by $\operatorname{LoxP}(\mathrm{L})$ sites to yield a so-called Lox-STOP-Lox ( $L S L)$ allele. In the presence of Cre recombinase, the STOP element is excised, allowing expression of the oncogene from its endogenous promoter. When Cre recombinase is introduced in a pancreas-specific fashion by crossing with $\mathrm{Pd} x 1$-Cre transgenic animals, $100 \%$ of the mice develop PanIN lesions that gradually progress, such that a significant proportion of mice develop PDA, although malignancy appears late at approximately 12 months of age.

In order to test whether ICMT deficiency would delay or inhibit the progression of PanINs, we crossed the Pdx 1 -Cre;LSL-Kras ${ }^{G 12 D}$ mice with mice carrying the floxed allele of $\operatorname{Icmt}\left(\operatorname{Icm} t^{f l x} / f x\right)$. We generated mice with the experimental genotype Icmt $t^{f l x} / f x ; P d x 1-C r e ; L S L-K r a s^{G 12 D}$ and littermate control animals with the genotype Icmt ${ }^{f l x /+} ; P d x 1$ Cre;LSL-Kras ${ }^{G 12 D}$, which retained a wild-type copy of Icmt, even in the presence of Cre. Mice of both genotypes were born at the expected Mendelian ratios and gained weight normally. Animals of both genotypes were sacrificed at intervals following birth and subjected to necropsy. PCR of genomic DNA from the pancreas, liver, and spleen confirmed recombination of the floxed alleles Icm $t^{f l x /+}, I c m t^{f l x / f l x}$, and $L S L-\operatorname{Kras}^{G 12 D}$ in the pancreas but not in other tissues (not shown). 

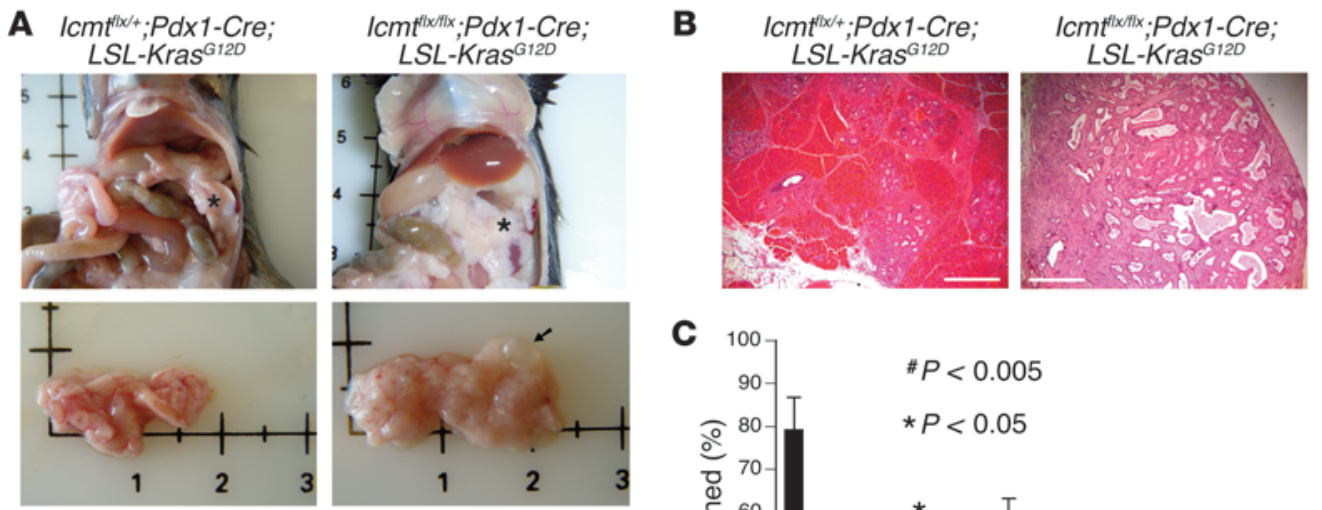

C
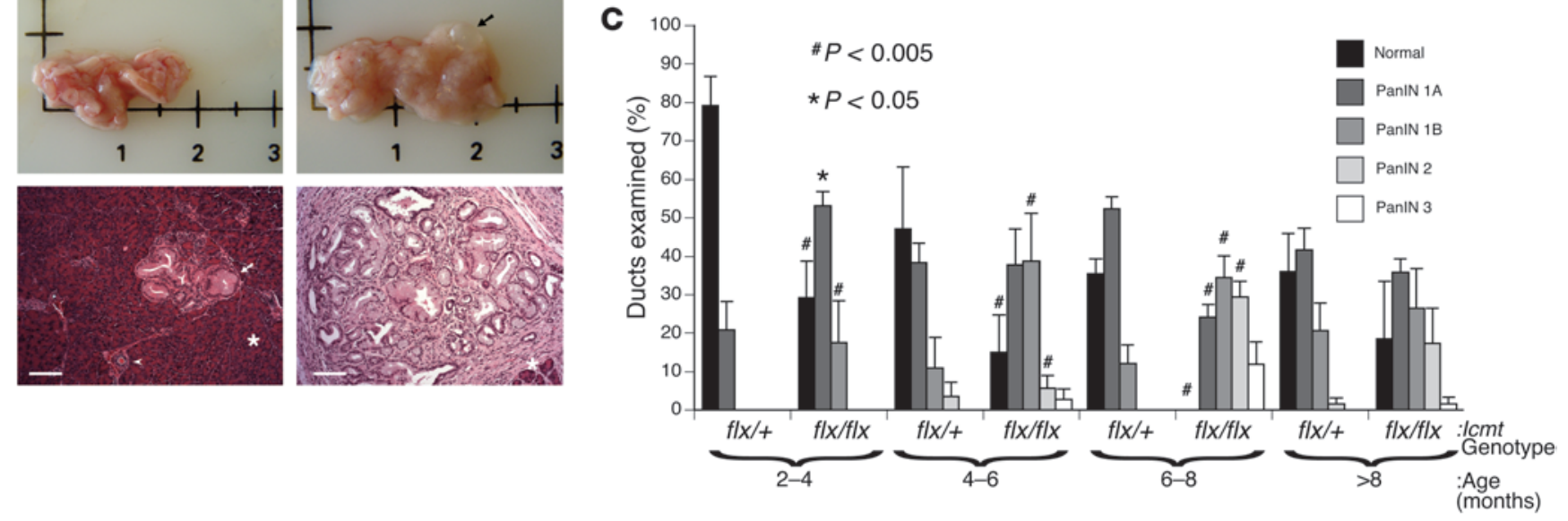

Figure 1

ICMT deficiency exacerbates PanIN lesions in Pdx1-Cre;LSL-Kras ${ }^{G 12 D}$ mice. (A) Pancreatic pathology of representative 4-month-old mice of the indicated genotypes. Top panels show the gross specimen; asterisks indicate the pancreas in situ and rulers show centimeter marks. Middle panels: The ICMT-deficient pancreas to the right is larger, with fibrotic nodules and a cyst (arrow). Lower panels show H\&E-stained sections from the same pancreata.

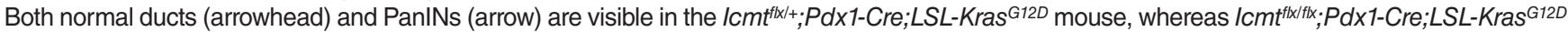
mice have more extensive PanINs. Normal acinar tissue (asterisk) is scant in the ICMT-deficient mice. Scale bars: $100 \mu \mathrm{m}$. (B) H\&E-stained sections of pancreata from 8-month-old animals of the indicated genotypes reveal more extensive PanIN lesions in the ICMT-deficient animals (right), in which most of the acinar tissue is replaced by PanINs and fibrosis. Scale bars: $500 \mu \mathrm{m}$. (C) Quantification of the percentage of normal ducts, PanIN-1A, 1B, 2, and

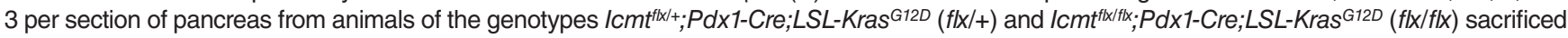
at the indicated ages. Data shown are the mean \pm SEM from at least four animals of each age and genotype.

The pancreata of Icmt $f^{l x / f l x} ; P d x 1-C r e ; L S L-K r a s^{G 12 D}$ mice appeared grossly abnormal (Figure 1A). They were larger and more fibrotic than pancreata from age-matched controls $(1.24 \pm 0.15 \mathrm{~g}$ vs. 0.79 \pm 0.12 g at 8 months; $n=13 ; P<0.01)$ and frequently showed cysts (Figure 1A). Strikingly, the pancreata of 2- to 3-monthold mice of the experimental genotype were found to contain vastly more PanIN lesions than control animals (Figure 1, A and $\mathrm{C}$, and Supplemental Figure 3A). Indeed, by 6 months of age, almost all of the normal pancreatic architecture was replaced by abnormal ducts and PanINs in Icmt flx $f l x_{2} ; P d x 1$-Cre;LSL-Kras ${ }^{G 12 D}$ mice, whereas, despite numerous PanINs, areas of normal ducts and acini were readily apparent in $I c m t^{f x /+} ; P d x 1-C r e ; L S L-K r a s^{G 12 D}$ mice (Figure 1B). Analysis of the histological grade of PanINs (23) revealed that the lesions progressed more rapidly to more advanced stages in Icmt $t^{f l x / f l x} ; P d x 1$-Cre; LSL-Kras ${ }^{G 12 D}$ animals than in Icm $t^{f l x /+} ; P d x 1-C r e ; L S L-K r a s^{G 12 D}$ animals (Figure 1C). Thus, in addition to increasing the number of PanINs, ICMT deficiency accelerated the progression to more advanced stages of disease.

ICMT deficiency alone is insufficient to induce pancreatic neoplasia. We observed no evidence of PanIN development or other neoplastic change in the pancreata of ICMT-deficient animals that did not express KRAS ${ }^{\mathrm{G} 12 \mathrm{D}}$ (Supplemental Figure 1B), indicating that the effects of ICMT deficiency depend on oncogenic Kras. Inactivation of the tumor suppressor $\mathrm{p} 53$, for example, with a dominant-negative $p 53^{R 172 H}$ allele, dramatically accelerates many oncogene-driven cancers, including KRAS ${ }^{\mathrm{G} 12 \mathrm{D}}$-driven PDA in mice (24). Like Icmt $t^{l x} / f t x ; P d x 1$ -
Cre mice, Icm $t^{f x / f l x} ; P d x 1$-Cre;LSL-p53 $3^{R 172 H}$ animals failed to develop any pancreatic neoplasm (Supplemental Figure 2). Thus, ICMT deficiency was incapable of inducing pancreatic neoplasia in the absence of oncogenic KRAS, even when p53 was inactivated.

ICMT deficiency increases the incidence of KRAS $12 D$-induced acinarductal metaplasia in the pancreas. The virtual replacement of normal pancreatic architecture with PanINs observed in 6-month-old Icm t ${ }^{f l x} / f l x ; P d x 1-C r e ; L S L-K r a s^{G 12 D}$ mice suggested a dramatic proliferation of ductal epithelial cells combined with a loss of acinar cells, which may occur by a process known as acinar-ductal metaplasia (ADM). ADM has been associated with PanIN lesions in human pancreatic samples as well as in the early changes seen in KRAS $^{\mathrm{G} 12 \mathrm{D}}$-driven models of PDA (25). To detect ADM, we stained frozen sections of the pancreata from control and experimental 2-month-old animals using antibodies against the acinar cell marker amylase and the ductal cell marker cytokeratin-19 (CK19) (Figure 2A). In the pancreata from Icmt ${ }^{f l x /+} ; P d x 1-C r e ; L S L-K r a s^{G 12 D}$ control mice, the cells staining positive for CK19 were negative for amylase and vice versa. This is likely because ADM is a transitory stage occurring at a low frequency in these pancreata and therefore was not captured in the sections examined. In contrast, PanIN lesions in the pancreata of Icmt $f^{f x / f l x} ; P d x 1-C r e ; L S L-K r a s^{G 12 D}$ mice revealed clusters of cells that stained for both markers, indicating higher levels of ADM (Figure 2, A and B). H\&E staining of these pancreata revealed areas where ducts appeared to be developing from acinar tissue, consistent with ADM (Figure 2C). 
A
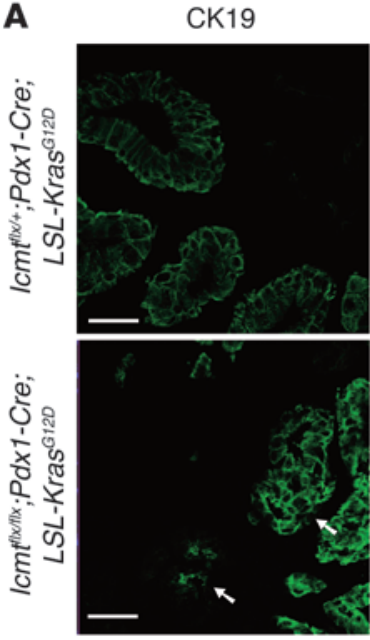

B

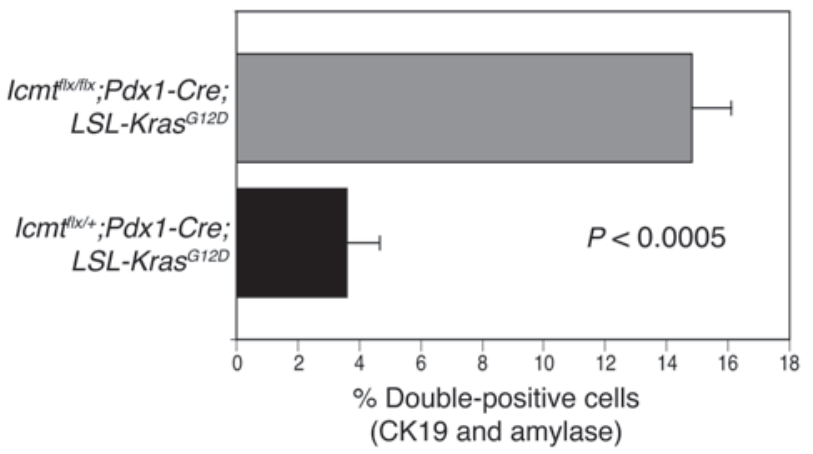

Amylase
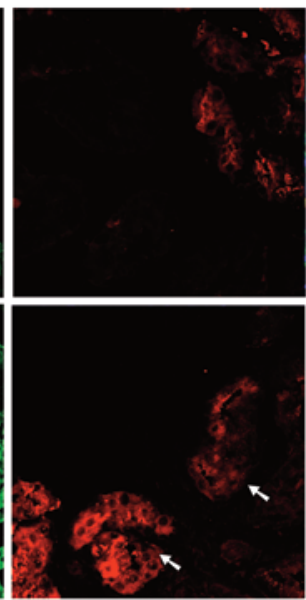

$\mathbf{K}$
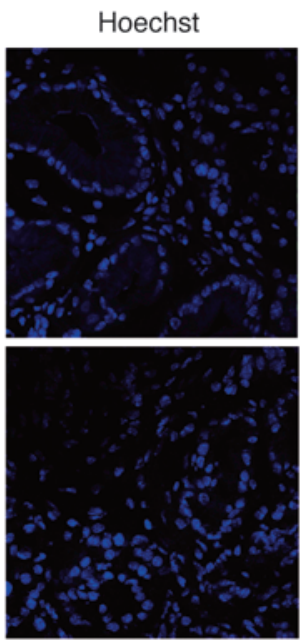
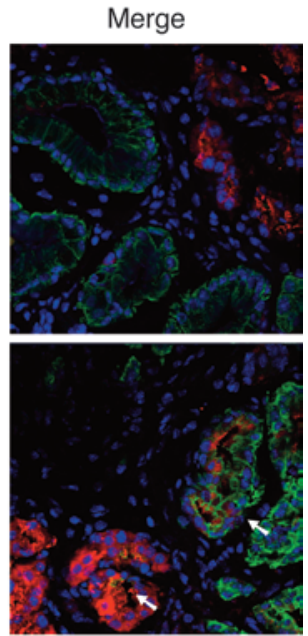

C

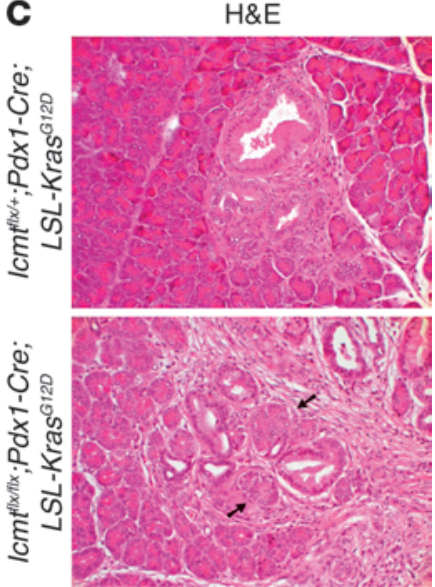

\section{Figure 2}

Acinar-to-ductal metaplasia contributes to PanINs in ICMT-deficient pancreata. (A) Frozen sections of pancreata from 2-month-old mice of the indicated genotypes were stained by immunofluorescence for cytokeratin 19 (CK19, a ductal marker, green) and amylase (an acinar marker, red) and counterstained with Hoechst (blue) to mark nuclei. Whereas staining in $I c m t^{f / x /+; P d x 1-C r e ; L S L-K r a s}{ }^{G 12 D}$ pancreata revealed strict segregation of cells that stained for each marker, some cells associated with PanINs in Icmt ${ }^{f f x} / f x ; P d x 1-C r e ; L S L-K r a S^{G 12 D}$ pancreata stained for both markers (arrows). Scale bars: $50 \mu \mathrm{m}$. (B) Percentage of cells positive for both amylase and CK19 in five low-power fields examined for each genotype (mean \pm SEM, $n=3, P<0.05$ ). (C) H\&E-stained sections of pancreata from the same mice showing PanINs. The PanINs in the ICMT-deficient pancreas more frequently revealed areas in which acinar cells appeared to be differentiating into ductal cells (arrows).

ICMT deficiency worsens the fibrosis and inflammation associated with Kras ${ }^{G 12 D}$-induced PanIN development. Abundant desmoplastic stroma is one of the hallmarks of PDA (26). The desmoplastic stroma consists of ECM, activated fibroblasts, inflammatory cells, and neovasculature. Desmoplastic stroma is seen in human tumors and is associated with PanINs in pancreata from Pdx1-Cre;LSL-Kras ${ }^{G 12 D}$ mice (11). We observed that the pancreata of Icmt $t^{f x / f l x} ; P d x 1-C r e ; L S L-K r a s^{G 12 D}$ mice were larger and more fibrotic than age-matched Icm $f^{f x / f x} ; P d x 1$ Cre;LSL-Kras ${ }^{G 12 D}$ animals. Consistent with this observation, sections of pancreata from 2-month-old mice stained with Masson's trichrome revealed deposition of collagen (Figure 3, A and B), which is the hallmark of fibrosis (27), and were positive for $\alpha$-smooth muscle actin, a marker of activated pancreatic stellate cells (Supplemental Figure 3B). Strikingly, while pancreata from Icmt ${ }^{f x /+} ; P d x 1-C r e ; L S L-$ $\operatorname{Kras}^{G 12 D}$ mice stained positive for collagen and activated pancreatic stellate cells in the immediate vicinity of the PanIN lesions, pancre- ata from Icm thx/flx; $P d x 1$-Cre;LSL-Kras ${ }^{G 12 D}$ mice revealed a much higher degree of fibrosis with substantial areas of collagen stretching throughout the organ (Figure 3, A and B, and Supplemental Figure $3 \mathrm{~B})$. To determine whether the enhanced deposition of collagen was also associated with an increased inflammatory infiltrate, we stained frozen sections of pancreata from the same animals with antibodies directed against the panleukocytic marker CD45. The ICMT-deficient pancreata showed markedly increased staining for CD45 compared with the KRAS ${ }^{\mathrm{G} 12 \mathrm{D}}$-expressing pancreata that also expressed ICMT, indicating an enhanced inflammatory response in ICMT-deficient pancreata (Figure 3, C and D).

ICMT deficiency leads to partial mislocalization of KRAS in pancreatic cells but does not affect KRAS-driven MAPK signaling or epithelial proliferation. We have previously shown that GFP-RAS fusion proteins are partially mislocalized from the plasma membrane to the cytosol and internal membranes in ICMT-deficient MEFs $(28,29)$. In 
A

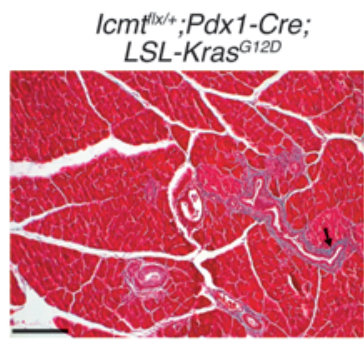

C
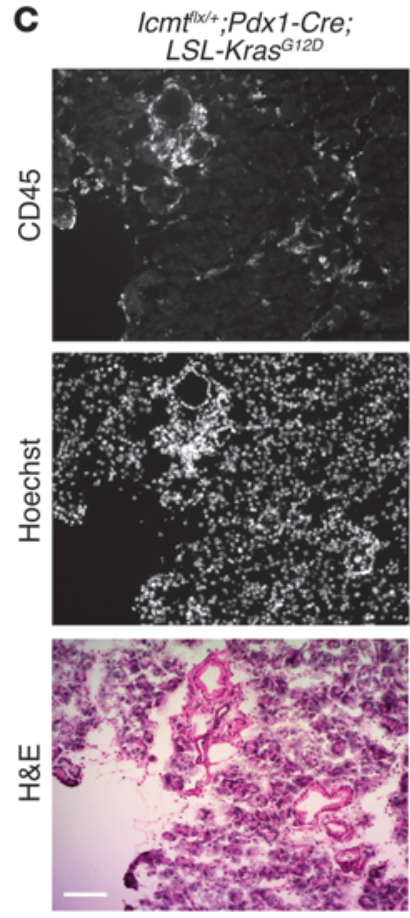
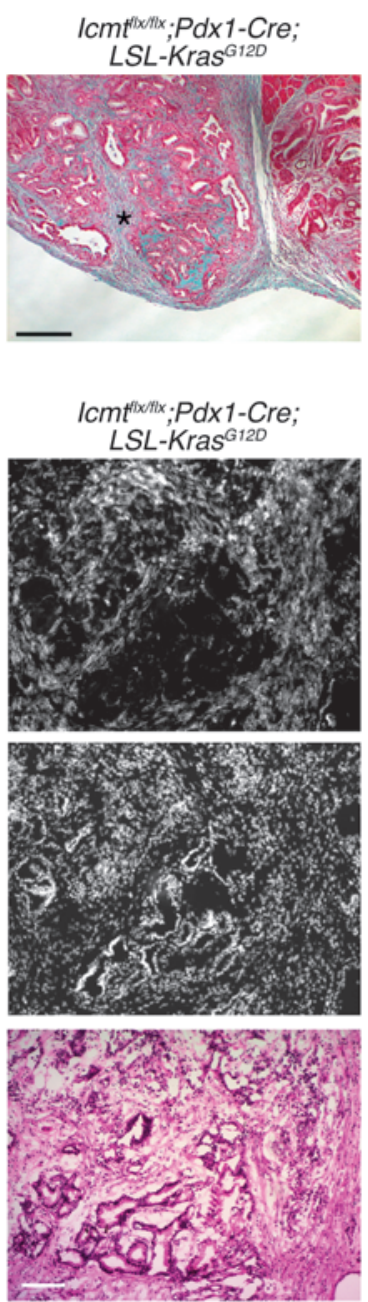

B

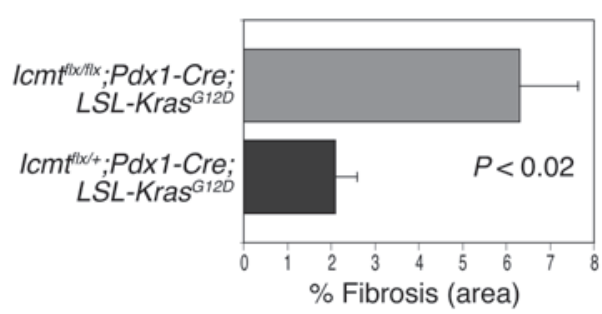

D

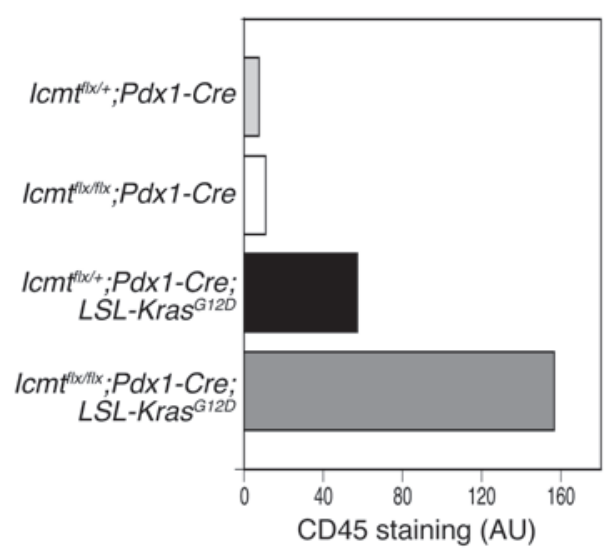

Figure 3

Accelerated fibrosis and leukocytic infiltration associated with PanINs in mice expressing oncogenic KRAS in the setting of ICMT deficiency. (A) Masson's trichrome staining of sections of fixed, paraffin-embedded pancreas from 2-month-old mice of the indicated genotypes. The arrow indicates fibrosis surrounding PanIN in pancreas from an $I c m t^{f|l|+; P d x 1-C r e ; L S L-K r a s}{ }^{G 12 D}$ mouse, while the asterisk indicates more extensive fibrosis in ICMT-deficient pancreas. Scale bars: $200 \mu \mathrm{m}$. (B) Percentage of fibrotic areas in five low-power fields $($ mean \pm SEM, $n=8, P<0.05)$. (C) Frozen sections of pancreata from 2-month-old mice of the indicated genotypes stained by immunofluorescence for CD45 (leukocyte common antigen) and counterstained with Hoechst to mark nuclei. Also shown in the bottom panels are H\&E stains of adjacent frozen sections. Scale bars: $100 \mu \mathrm{m}$. (D) Quantification of the average amount of CD45 staining per frozen section (five sections per mouse). Data shown are the mean of two age-matched mice for each genotype.

order to confirm the mislocalization of KRAS in ICMT-deficient pancreata, we examined the distribution of endogenous KRAS in subcellular fractionations. Pancreata from 3-month-old Icm $t^{f l x /+}$; $P d x 1$-Cre or Icmt $t^{f x / f x} ;$ Pdx 1 -Cre mice were homogenized and fractionated. KRAS was found almost exclusively in the P100 membrane fraction of pancreata from Icm $t^{f x /+} ; P d x 1$-Cre mice, consistent with previous reports (29). In contrast, KRAS was evenly distributed between the P100 and S100 fractions from the homogenates of Icmt $t^{f x} f f x ; P d x 1$-Cre pancreata (Figure 4A). Indeed, the relative content of KRAS in the cytosol (S100) increased 7-fold in ICMT-deficient pancreata relative to control.

The observation that oncogenic KRAS does not require methylation by ICMT to induce PanINs suggests that KRAS signaling is intact in ICMT-deficient pancreatic cells, despite partial mislocalization to the cytosol. To confirm this conclusion, we studied
ERK activation in PanIN lesions. Upregulation of nuclear phospho-ERK (pERK) staining downstream of KRAS ${ }^{\mathrm{G} 12 \mathrm{D}}$ expression is an early feature of mouse PanIN lesions, whereas normal pancreatic tissue is negative for pERK staining $(30,31)$. We observed similar levels of staining for pERK by immunohistochemistry in early PanIN lesions in pancreata from both $I c m t^{f x /+} ; P d x 1$ Cre;LSL-Kras ${ }^{G 12 D}$ and Icm $t^{f x / f f l x} ; \mathrm{Pdx} 1-\mathrm{Cre} ; \mathrm{LSL}-\mathrm{Kras}{ }^{G 12 D}$ mice (Figure $4 \mathrm{~B})$. This suggests that KRAS ${ }^{\mathrm{G} 12 \mathrm{D}}$ stimulates the MAPK pathway in the pancreas even in the absence of ICMT and is consistent with the observation that, while silencing ICMT in HeLa cells slightly diminishes GTP-loading of RAS and inhibits pERK by 25\%, it does not eliminate RAS/MAPK signaling (Supplemental Figure 4). Concordant with the observation that KRAS-stimulated MAPK signaling was preserved in ICMTdeficient pancreata, the PanIN lesions in the pancreata of both 
A
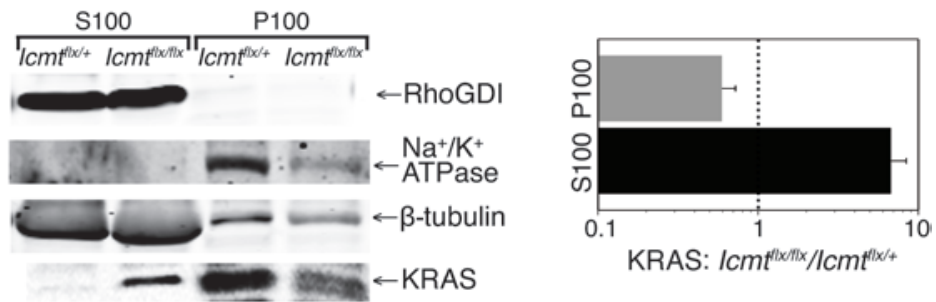

B
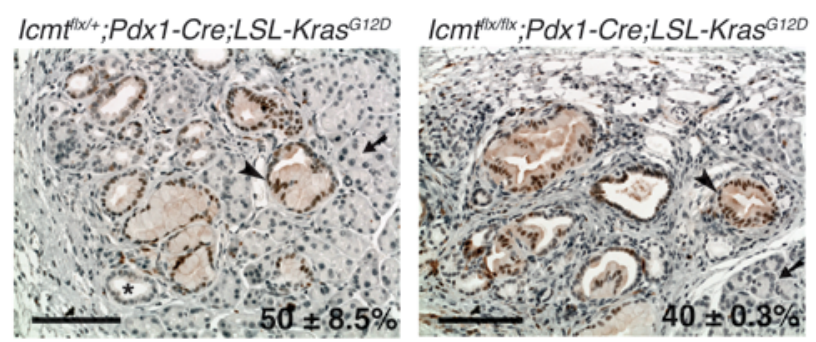

C
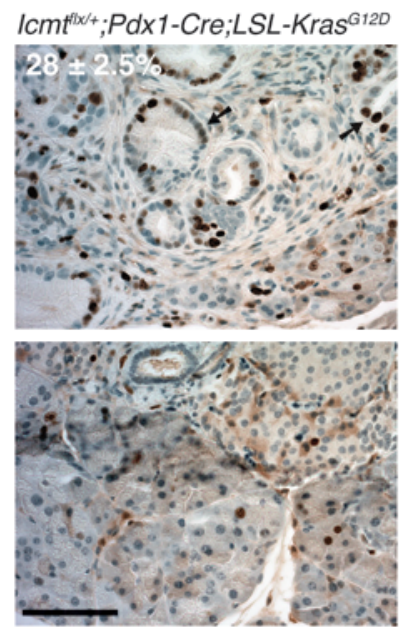

\section{Figure 4}

ICMT deficiency mislocalizes KRAS in pancreatic cells but does not inhibit ERK activation or proliferation. (A) Membrane association of endogenous KRAS in subcellular fractionations of homogenized pancreas from 3-month-old $/ \mathrm{cm} t^{f(x /+;} ; P d x 1$-Cre

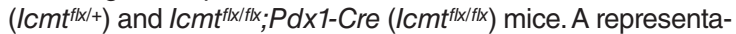
tive immunoblot of KRAS in cytosolic (S100) and membrane (P100) fractions is shown, as is a quantification of three such experiments, the results of which are shown as the mean \pm SEM of the ratios $\left(\mathrm{Icmt} / \mathrm{fl} / \mathrm{fl} / \mathrm{l} / \mathrm{cm} \mathrm{t}^{f / x /+}\right.$ ) of immunodetected KRAS in P100 and S100 fractions normalized to the amount of $\beta$-tubulin present in each fraction. $\mathrm{Na}^{+} \mathrm{K}^{+}$-ATPase and RhoGDI were blotted to assess the purity of the membrane versus cytosolic fractions. (B) Immunohistochemical staining for pERK in paraffin sections of pancreata from 3-month-old mice of the indicated genotypes. Whereas normal ducts (asterisk) and acinar cells (arrows) were negative for pERK, early PanIN lesions showed strong nuclear and cytoplasmic staining (arrowheads) in pancreata from both genotypes, indicating that MAPK activation is unaffected by ICMT deficiency. (C) Immunohistochemical staining for Ki67 in paraffin sections of pancreata from 2-month-old mice of the indicated genotypes. Upper panels show areas of PanIN lesions, and lower panels show normal ducts. The nuclei of ductal cells in PanINs stained for Ki67 in both genotypes (arrows). Scale bars: $100 \mu \mathrm{m}$ (B and C). Percentage of nuclei positive for pERK or Ki67 in 500 PanIN-associated cells examined (mean \pm SEM, $n=3$, NS).
$I_{c m} t^{f l x /+} ; P d x 1-C r e ; L S L-K r a s^{G 12 D}$ and Icm $t^{f l x / f l x} ; P d x 1-C r e ; L S L-K r a s^{G 12 D}$ mice stained for Ki67 to similar degrees (Figure 4C), indicating similar levels of proliferation in both genotypes.

ICMT deficiency does not significantly affect the survival of mice expressing oncogenic Kras ${ }^{G 12 D}$ and dominant-negative $p 53^{R 172 H}$ in the pancreas. The progression of PanINs to invasive, metastatic PDA in the LSL-Kras ${ }^{G 12 D}$;Pdx1-Cre mouse model occurs at low penetrance and with a latency of close to 1 year (11). In order to determine whether the acceleration of PanIN progression in Icm t $t^{f x / f l x} ; L S L-K r a s^{G 12 D} ; P d x 1$ Cre mice was associated with increased PDA, we examined pancreata from $I c m t^{f l x /+} ; L S L-K r a s^{G 12 D} ; P d x 1-C r e$ and Icm $t^{f l x / f l x} ; L S L-K r a s^{G 12 D}$; $P d x 1$-Cre mice sacrificed at various ages. The Icmt ${ }^{f l x} / f l x ; L S L-K r a s^{G 12 D}$; Pdx1-Cre mice showed an increased percentage of areas of PDA compared with controls at each age, with the earliest observed case occurring at 5.7 months of age versus 7.7 months in the control animals (Figure 5A). Two mice from each genotype were found to have metastases in the liver at the time of necropsy (Figure 5B).

The long latency of PDA in both genotypes made survival studies challenging. We therefore sought to accelerate the progression to PDA by inactivating a relevant tumor suppressor. $p 53^{R 172 H}$ has been shown to accelerate the progression to PDA in the LSL-Kras ${ }^{G 12 D}$;Pdx1-Cre model and to decrease survival to 5 months (24). We therefore bred an $L S L-p 53^{R 172 H}$ allele into our mice, generating Icmt $t^{f l x f f l x} ; L S L-K r a s^{G 12 D} ; P d x 1-C r e ; L S L-p 53^{R 172 H}$ and Icmt $t^{f l x /+} ; L S L-K r a s^{G 12 D} ; P d x 1-C r e ; L S L-p 53^{R 172 H}$ animals, which were followed until they succumbed or showed signs of significant illness. ICMT deficiency decreased survival, but the magnitude of the effect was small and did not reach statistical significance (Figure 5C). At necropsy, mice from both genotypes were found to harbor large primary tumors in the pancreas, with frequent metastatic lesions in the liver (Figure 5D).

ICMT deficiency in the pancreas does not increase susceptibility to cerulein-induced inflammation. The exacerbation of PanIN lesions in Icm t flxflx $; P d x 1$-Cre; LSL-Kras ${ }^{G 12 D}$ mice in the setting of unaffected RAS/MAPK signaling suggests that ICMT deficiency may affect disease progression through a non-cell-autonomous mechanism. Chronic pancreatitis is a significant risk factor for PDA in humans (32). Because the accelerated PanINs observed in ICMT-deficient pancreata were associated with an inflammatory infiltrate (Figure 3, C and D), we tested the hypothesis that the accelerated KRAS ${ }^{G 12 D}$-induced PanIN development seen in mice lacking ICMT in the pancreas was due either to an increased susceptibility to inflammation or to a failure to efficiently resolve an inflammatory insult. To test this hypothesis, we used cerulein-induced pancreatitis because it is the best-studied mouse model of inflammation in this organ. Cerulein is an analog of cholecystokinin 

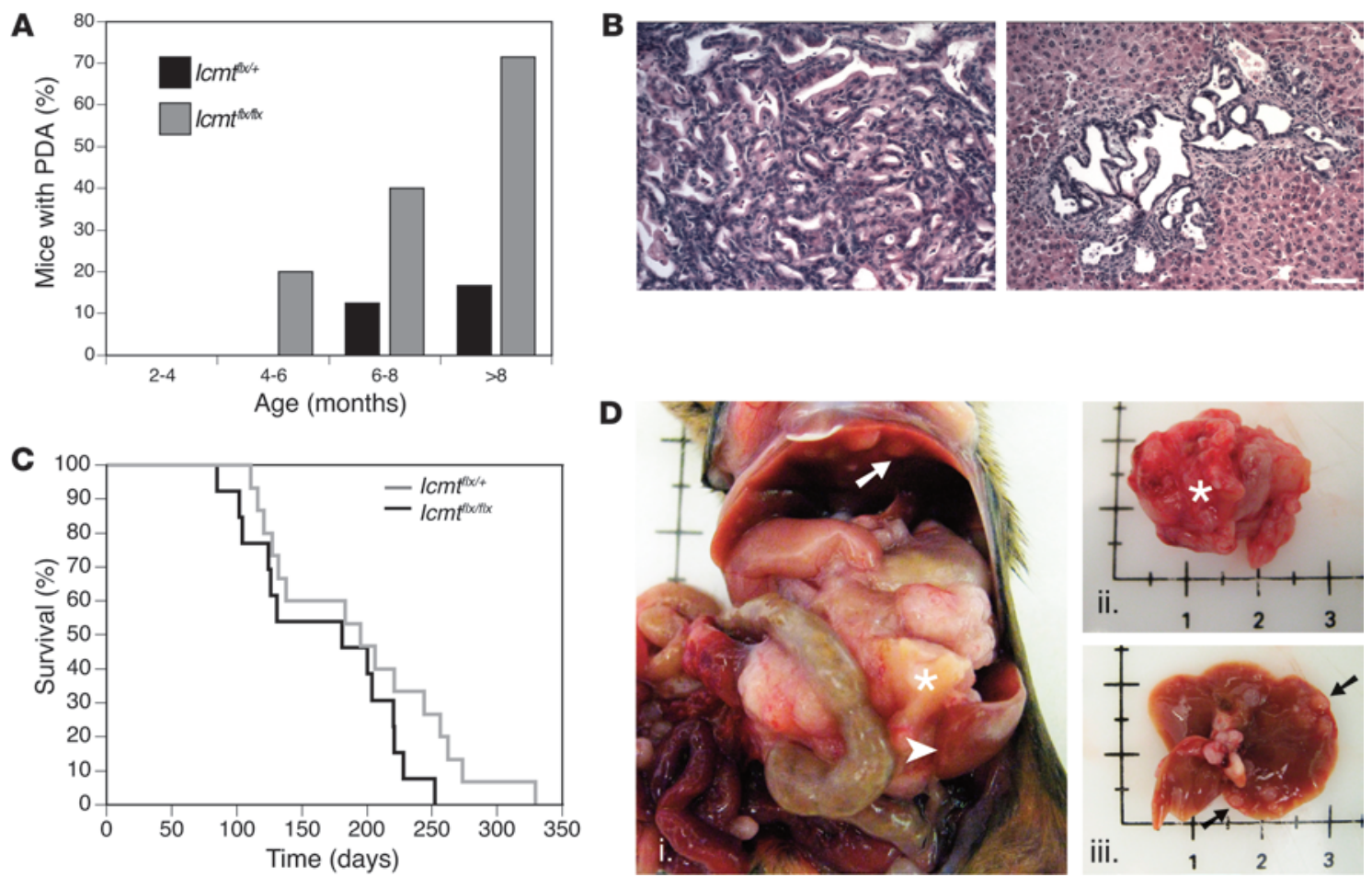

\section{Figure 5}

ICMT deficiency accelerates the development of PDA but does not significantly affect the survival of mice expressing oncogenic KRASG12D and

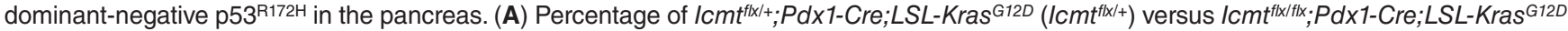
$\left(I c m t^{f / x / f l}\right)$ mice of the indicated ages that had PDA upon histological examination of the pancreas at necropsy $($ cohort size $=17)$. $(B)$ H\&E staining of paraffin sections of the pancreas (left) and liver (right) of a 6-month-old Icmt $t^{f / x} / f x ; P d x 1$-Cre; LSL-Kras ${ }^{G 12 D}$ mouse that developed metastatic

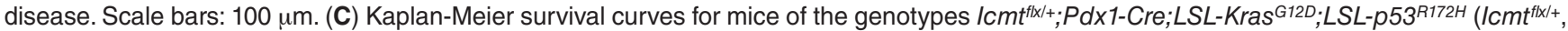

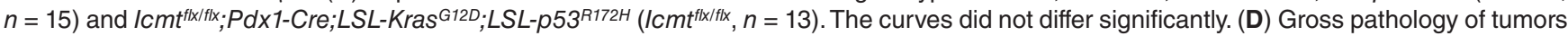
found in a 6-month-old $/ c m t^{f|x|+; P d x 1-C r e ; L S L-K r a s}{ }^{G 12 D} ; L S L-p 53^{R 172 H}$ mouse. Clearly visible are a large tumor at the head of the pancreas (asterisk), liver metastases (arrows), and an enlarged spleen (arrowhead).

that, when administered to rodents in supraphysiologic doses, stimulates premature intracellular activation of pancreatic digestive enzymes that cause tissue damage resulting in pancreatitis $(33,34)$. Icmt ${ }^{f l x / f l x} ; P d x 1$-Cre and $I c m t^{f l x /+} ; P d x 1$-Cre mice were treated with 7 hourly i.p. injections of cerulein over a period of 2 days to induce acute pancreatitis (35). No difference was observed in the degree of inflammation observed in the two genotypes (Supplemental Figure 5A). By day 7 after cerulein administration, the pancreata showed almost complete resolution of inflammation. Again, there was no difference observed with or without pancreatic ICMT (Supplemental Figure 5A). Similar results were obtained when a model of chronic pancreatitis was employed in which mice were treated with 7 hourly cerulein injections, thrice weekly, for 3 weeks (Supplemental Figure 5B). Thus, ICMT deficiency in the pancreas affects neither cerulein-induced pancreatitis nor the resolution of the inflammatory condition.

ICMT-deficient pancreatic ductal cells proliferate more rapidly and are resistant to senescence. We next sought evidence for a cell-autonomous effect of ICMT deficiency. In order to determine whether pancreatic ductal epithelial cells (PDECs) might have a growth advantage when ICMT deficient, we isolated PDECs from the main pancreatic duct from 2-month-old mice of the genotypes Icmt $t^{f l x / f l x} ; L S L-K r a s^{G 12 D}, I c m t^{f l x /+} ; L S L-K r a s^{G 12 D}, I c m t^{f l x} f f l x$, and $I c m t^{f l x /+}$ and cultured them in vitro, embedded in $3 \mathrm{D}$
Matrigel culture, where they were grown as spheroids (ref. 36 and Figure 6A). To induce recombination of the LoxP sites, the ductal cells were infected with adenovirus-directing expression of Cre recombinase (Adeno-Cre). The efficiency of recombination was confirmed by PCR analysis of genomic DNA isolated from the infected ductal cells (Figure 6B). The PDECs harvested from the Matrigel were replated on conventional tissue culture plastic (2D culture) for 13 days, and the rates of proliferation were measured with an MTS assay. Interestingly, in the absence of oncogenic KRAS, ICMT-deficient PDECs grew faster (Figure 6C). As expected, the growth rate of KRAS ${ }^{\mathrm{G} 12 \mathrm{D}}$-transformed PDECs was markedly increased relative to that of untransformed cells expressing ICMT, and ICMT deficiency slightly increased the proliferative rate of KRAS ${ }^{\mathrm{G} 12 \mathrm{D}}$-transformed PDECs (Figure 6D). Thus, contrary to expectations, ICMT deficiency increased the growth rate of PDECs independently of RAS transformation.

When shifted from 3D to 2D culture, wild-type PDECs undergo premature senescence after approximately 7 days, whereas PDECs expressing KRAS ${ }^{\mathrm{G} 12 \mathrm{D}}$ do not (37). Senescence is associated with increased expression of the cell cycle inhibitor $\mathrm{p} 16^{\mathrm{INK} 4 \mathrm{~A}}$. PDECs were harvested after 1 and 8 days in 2D culture for analysis of relevant proteins by Western blotting. Parallel samples of cells were fixed after 12 days in $2 \mathrm{D}$ culture and stained for expression of senescence-associated $\beta$-gal (SA $\beta$ gal). We observed that, while 
A
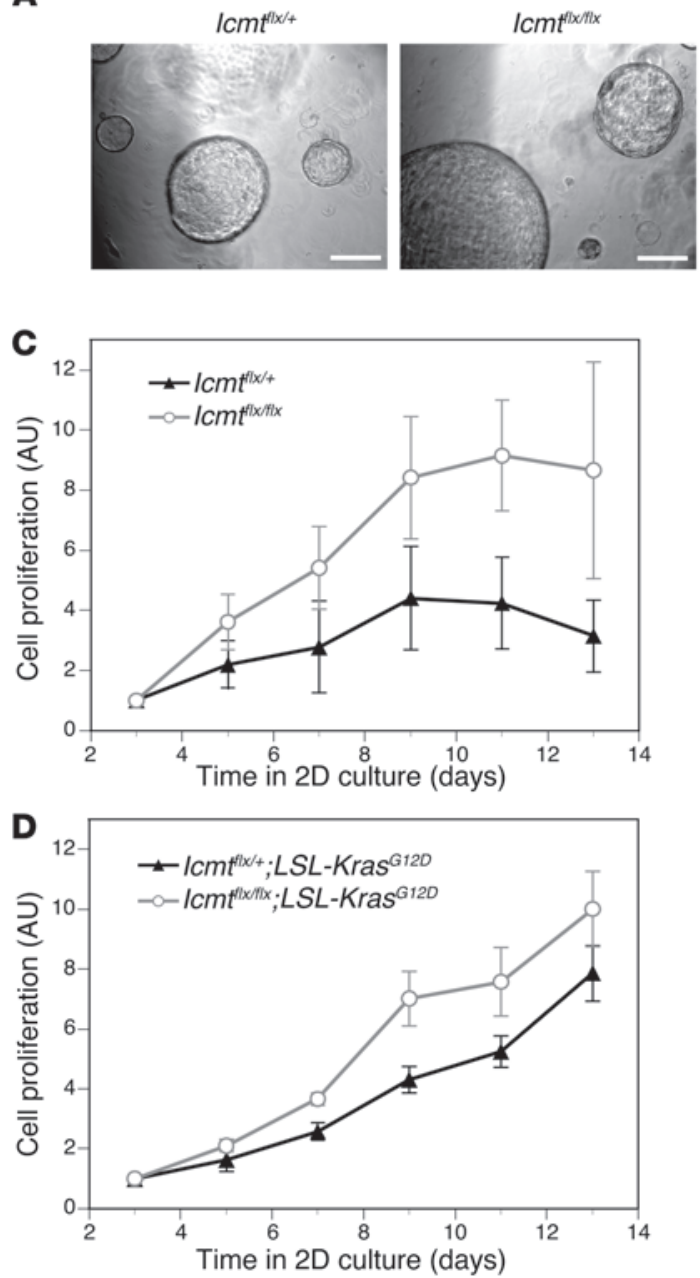

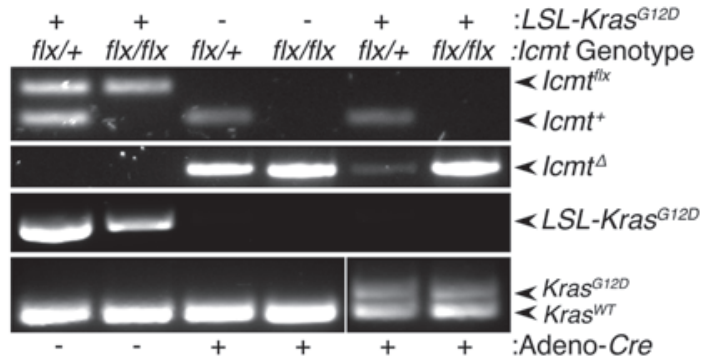

$\mathbf{E}$

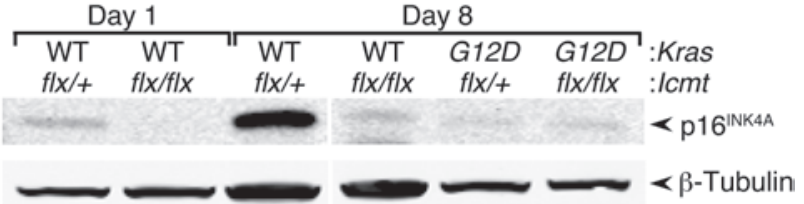

$\mathbf{F}$
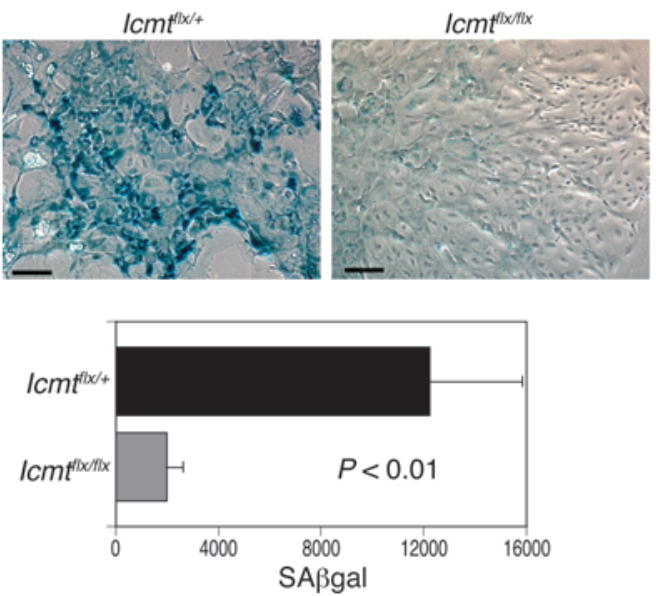

Figure 6

Cell-autonomous effects: ICMT-deficient PDECs are resistant to premature senescence in 2D culture. (A) Phase contrast micrographs of primary PDECs harvested from the main pancreatic duct of animals of the indicated genotypes and grown as spheroids in 3D Matrigel. Scale bars: 200 $\mu \mathrm{m}$. (B) Genotype analysis by PCR and agarose gel electrophoresis of DNA from PDECs of the indicated genotypes before and after initiation of LoxP recombination by infection with adenovirus-directed expression of Cre recombinase. (C and D) Proliferation as measured by MTS assay of PDECs of the indicated genotypes after switching from 3D to 2D culture on plastic. Values were normalized to the day-3 reading (value $=1$ ). Data are plotted as the mean \pm SEM; $n=4$ for $\mathbf{C}$ and $n=3$ for $\mathbf{D}$. (E) Immunoblots for $16^{\text {INK4A }}$ of lysates prepared from PDECs of the indicated genotypes on days 1 and 8 after switching from 3D to 2D culture. Blot shown is representative of three. (F) Phase contrast micrographs of PDECs of the indicated genotypes stained with X-gal for SA 3 gal 12 days after switching to 2D culture. Scale bars: $100 \mu \mathrm{m}$. Quantification of X-gal staining normalized to cell number is plotted as the mean \pm SEM in the graph below $(n=4, P<0.01)$.

PDECs from Icmt $t^{f l x /+}$ mice infected with Adeno-Cre expressed high levels of $16^{\text {INK4A }} 8$ days after shifting from 3D to $2 \mathrm{D}$ culture, PDECs from Icmt $f^{f l} / f l x$ mice did not (Figure 6E). Moreover, on day 12, while PDECs from Icm $t^{f x /+}$ mice showed strong SA $\beta$ gal expression (Figure 6F) indicating senescence and consistent with the lack of proliferation after 7 days (Figure 6C), PDECs from $I c m t^{f l x / f l x}$ mice showed markedly reduced SA $\beta$ gal expression (Figure $6 \mathrm{~F}$ ), concordant with low $\mathrm{p} 16^{\mathrm{INK} 4 \mathrm{~A}}$ expression. Thus, ICMT deficiency accelerates the growth of PDECs in tissue culture and allows them to escape premature senescence.

ICMT deficiency phenocopies Notch1 deficiency. Exacerbation of pancreatic neoplasia without evidence of altered RAS/MAPK signaling suggested that ICMT deficiency may accelerate the disease by affecting another signaling pathway that is independent of RAS, a possibility that is all the more plausible considering that RAS is but one of many substrates for ICMT. One clue to the involvement of another pathway came from a prominent extrapancreatic tumor observed in Icmt ${ }^{f l x} / f x ; P d x 1$-Cre; LSL-Kras ${ }^{G 12 D}$ mice. Pdx1-Cre has been shown to be leaky in that it is expressed in the epidermis as well as in the pancreas (16). This observation likely explains the cutaneous papillomas that were reported with low penetrance by Hingorani et al. in the initial description of the Pdx1-Cre;LSL-Kras ${ }^{G 12 D}$ model (11). We observed a dramatic increase in the frequency of papillomas on the faces of $I c m t^{f l x / f l x} ; P d x 1-C r e ; L S L-K r a s^{G 12 D}$ mice (Figure 7A). Fifty-nine percent of animals with this genotype developed these lesions compared with $7 \%$ of $I c m t^{f x /+} ; P d x 1-C r e ; L S L-K r a s^{G 12 D}$ mice. No lesions were observed on Icmt $t^{f l / f l x} ; P d x 1$-Cre mice, demonstrating that activated KRAS is necessary for these skin tumors. 

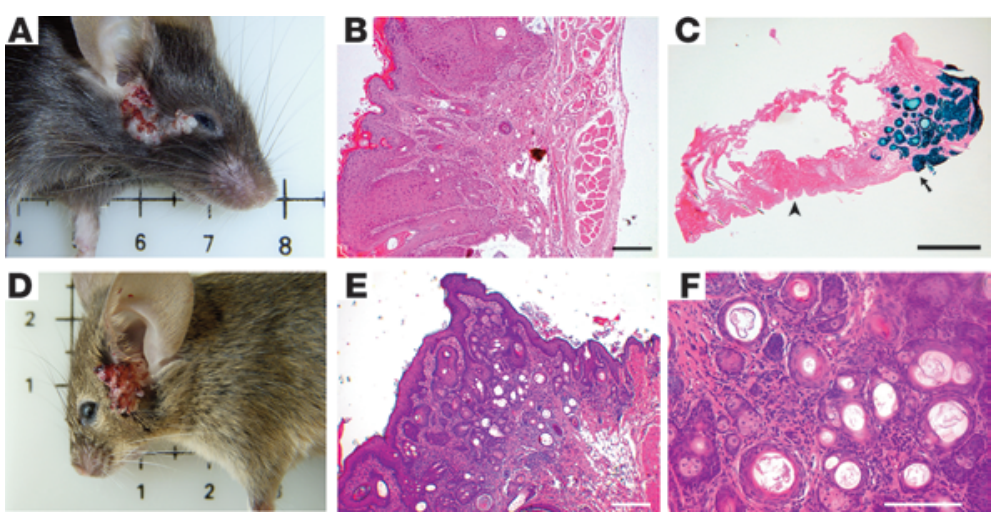

Figure 7

Epidermal tumors in Icmt $t^{f \mid x / f x} ; P d x 1-C r e ; L S L-K r a s^{G 12 D}$ mice. ICMT deficiency in conjunction with oncogenic Kras driven by $P d \times 1$-Cre expression in the epidermis leads to papillomas on the face that can progress to squamous cell carcinoma. (A) A 4-month-old Icmt ${ }^{f|x| f \mid x} ; P d x 1-C r e ; L S L-K r a s^{G 12 D}$ mouse manifested a facial papilloma between the eye and pinna. Fifty-nine percent of animals with this genotype developed these lesions compared with $7 \%$ of $I c m t^{f|l|+} ; P d x 1-C r e ; L S L-K r a s{ }^{G 12 D}$ mice. (B) H\&E staining showed the lesion to be consistent with a verruca vulgaris. Scale bar: $100 \mu \mathrm{m}$. (C) A papilloma from an Icmt ${ }^{f t / f f x} ; P d x 1-C r e ; L S L-K r a s^{G 12 D}$ mouse that also carried a Rosa26-LSL-LacZ Cre reporter allele was stained with X-gal to reveal Cre expression in the transformed (arrow) but not in the normal (arrowhead) epidermis. Scale bar: $500 \mu \mathrm{m}$. (D) A 3-month-old Icmt $t^{f \mid / f x} ; P d x 1$-Cre; LSL-Kras ${ }^{G 12 D}$ mouse showing a larger papilloma. (E and F) H\&E staining of the lesion shown in $\mathbf{D}$ revealed areas of malignant transformation into well-differentiated squamous cell carcinomas. Original magnification, $\times 10(\mathbf{E})$ and $\times 20(\mathbf{F})$. Scale bars: $100 \mu \mathrm{m}$.

Histologically, the lesions were consistent with verruca vulgaris (Figure 7B). We confirmed Pdx1-Cre expression in these lesions by crossing Icm $t^{f l x} / f x ; P d x 1-C r e ; L S L-K r a s^{G 12 D}$ mice with animals that harbor a Rosa26-LSL-LacZ allele (Figure 7C). Histopathological examination of some larger lesions revealed areas with an invasive growth pattern with cellular atypia, increased mitosis, and a high degree of keratinization with characteristic keratin "pearls" that are diagnostic of well-differentiated squamous cell carcinoma (Figure 7, D-F).

Notch 1 has been shown to act as a tumor suppressor both in mouse skin $(38,39)$ and in the pancreas of Pdx1-Cre;LSL-Kras ${ }^{G 12 D}$ mice (15). Indeed, the exacerbation of PanINs observed in Notch $1^{f l x / f l x}$; Pdx1-Cre;LSL-Kras ${ }^{G 12 D}$ mice (15) was identical to that observed in Icmt $t^{f l x / f l x} ; P d x 1-C r e ; L S L-K r a s^{G 12 D}$ mice. Importantly, in addition to accelerated PanINs, Notch $1^{f l x} f f x ; P d x 1$-Cre; $L S L-K r a s^{G 12 D}$ mice developed papillomas on the face and neck, particularly below the pinna, that were significantly larger and more frequent than those seen in Pdx1-Cre;LSL-Kras ${ }^{G 12 D}$ mice (16). Thus, ICMT deficiency phenocopied Notch1 deficiency both in the pancreas and epidermis of Pdx1-Cre;LSL-Kras ${ }^{G 12 D}$ mice. This observation suggests that ICMT and Notch1 function in the same pathway, which could be explained by a requirement of carboxyl methylation of an ICMT substrate for efficient Notch1 signaling.

ICMT is required for Notch signaling. To test the hypothesis that ICMT is required for efficient Notch 1 signaling, we used a coculture system that allows for stimulation of Notch1 in euploid U2OS human osteosarcoma cells with a relevant cell-surface ligand and quantification of Notch signaling with a luciferase reporter. We found that silencing ICMT with siRNA significantly decreased Notch1 signaling in response to fibroblasts expressing Jagged-2 (Figure 8A). Similar results were obtained when the same cells were stimulated with fibroblasts expressing Delta-1 (not shown).
To further test the hypothesis, we used D. melanogaster, a model genetic organism that is well-characterized with respect to the Notch pathway. We drove the expression of an shRNA targeting Drosophila ICMT, known as Ste14, in a tissue-specific fashion using the GAL4/ UAS system. UAS-shSte4 expressed in the developing wing using Ap-GAL4 (Figure 8B) or MS1096-GAL4 (Supplemental Figure 6) resulted in adult wings with vein defects identical to those seen in flies with Delta $(D l)$ loss-of-function alleles (40). Thus, ICMT deficiency in the developing wing phenocopies deficiency of a Notch ligand. Silencing of Icmt in the developing eye using GMR-GAL4 (Figure 8C) gave a rough eye phenotype not specific for, but consistent with, that observed in $\mathrm{Dl}$-mutant flies (40). To confirm the requirement of STE14 for Notch signaling in flies, we studied the expression of the Notch-dependent gene Cut (41) in the developing wing. We induced clones of cells expressing UAS-shSte14 that are marked by UAS-GFP by inducing stochastic expression of GAL4 with heat shock of early larvae. We examined the wing imaginal discs of third instar larvae for expression of CUT by immunofluorescence staining. CUT is normally expressed in a strip 3-4 cells wide at the dorsal-ventral boundary of the disc. Where the GFP-positive clone intersected the line of CUT staining, there was a marked decrease in the number of CUT-positive cells (Figure 8D and Supplemental Figure 7). Thus, as was observed in cultured human tumor cells, ICMT is required for efficient Notch signaling in Drosophila.

To further test the idea that inhibition of Notch signaling accounts for the effects of ICMT deficiency in the pancreas, we used compound E, a $\gamma$-secretase inhibitor that blocks Notch signaling (Figure $8 \mathrm{~A})$. We found that $\mathrm{p} 16^{\mathrm{INK} 4 \mathrm{~A}}$ expression in wild-type PDECs shifted from 3D to 2D culture was inhibited by a $\gamma$-secretase inhibitor (Supplemental Figure 8). Thus, Notch inhibition in these cells phenocopied ICMT deficiency with regard to $\mathrm{p} 16^{\text {INK4A }}$ expression. Because p16 $6^{\mathrm{INK} 4 \mathrm{~A}}$ expression is associated with cellcycle arrest, this observation is consistent with the mediation of senescence by ICMT-dependent Notch signaling and could explain the tumor-suppressor-like activity of ICMT in the Pdx1-Cre;LSLKras $^{G 12 D}$ model of pancreatic neoplasia.

Among the ways in which Notch 1 acts as a tumor suppressor in skin is through the negative regulation of $\mathrm{Wnt} / \beta$-catenin signaling, such that Wnt signaling is significantly upregulated in Notch1-deficient cells $(38,42)$. Interestingly, Wnt signaling is also markedly upregulated in the pancreas of Notch $1^{f l x / f l x}$; Pdx1-Cre;LSL-Kras ${ }^{G 12 D}$ mice (16). Staining for $\beta$-catenin in the PanIN lesions of Icmt $t^{f l x /+} ; P d x 1-C r e ; L S L-K r a s^{G 12 D}$ versus Icmt $t^{f l x / f l x}$; $P d x 1$-Cre; $L S L-K r a s^{G 12 D}$ mice revealed more $\beta$-catenin in the ductal epithelial cells of the latter genotype as well as a more cytoplasmic staining pattern characteristic of activated $\beta$-catenin (Supplemental Figure 9). Thus, Icm $t^{f l x / f l x} ; P d x 1-C r e ; L S L-K r a s^{G 12 D}$ mice also phenocopied Notch $1^{f l x / f l x} ; P d x 1^{-C r e} ; L S L-K r a s^{G 12 D}$ mice with respect to derepression of Wnt signaling.

\section{Discussion}

Enzymes that posttranslationally modify the CaaX motif of RAS have long been considered targets for anti-RAS drug discovery (43). The lack of success of FTIs as anticancer drugs does not invalidate this approach because FTIs failed due to alternative 
A

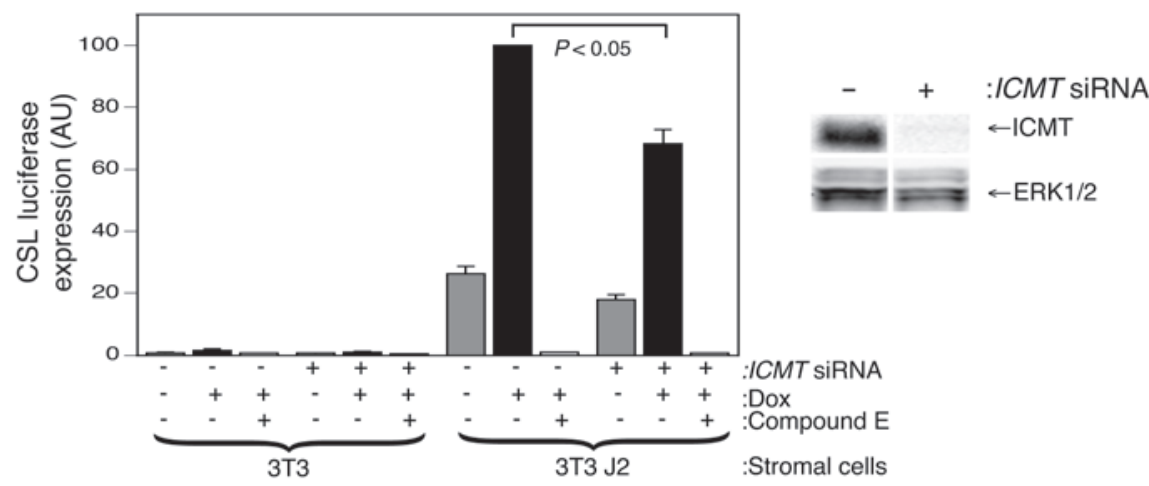

B

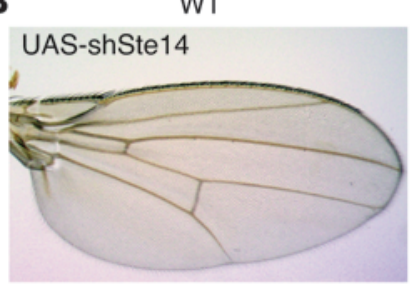

C

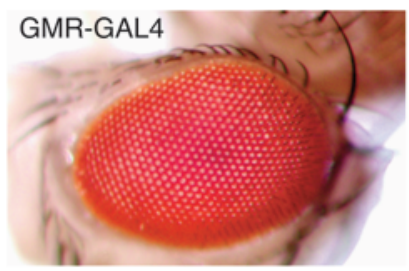

D

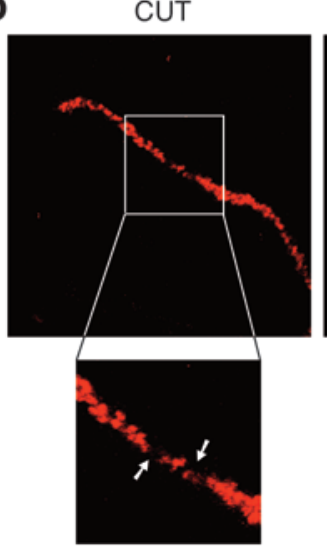

ICMT deficient

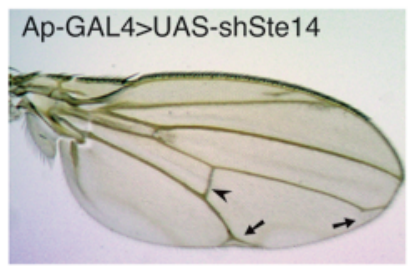

ICMT deficient

GMR-GAL4>UAS-shSte14

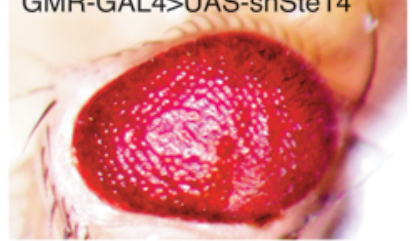

GFP

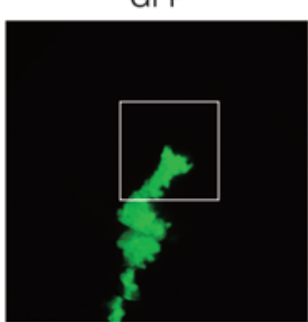

Hoechst

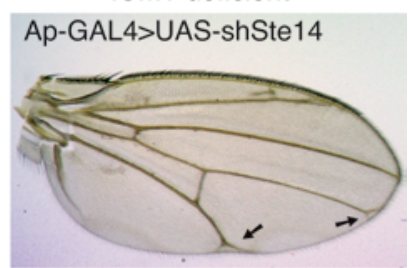

ICMT deficient

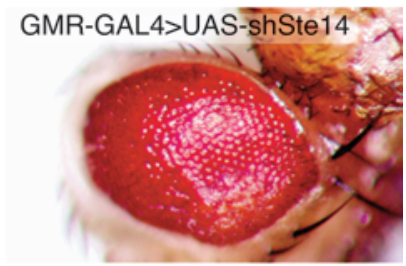

Merge
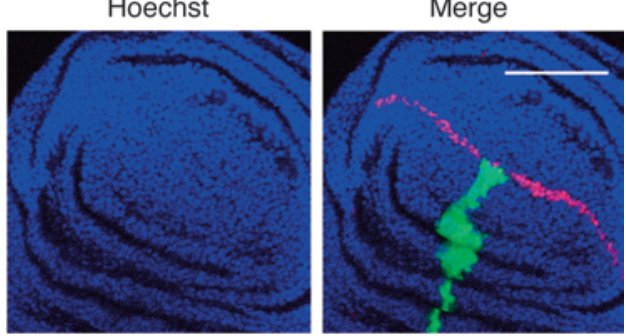

Figure 8

ICMT deficiency inhibits Notch1 signaling. (A) Notch signaling in mammalian cells quantified with a CSL firefly luciferase reporter. Shown are the ratios of CSL firefly/CMV Renilla in U2OS cells containing doxycycline-inducible FLAG-Notch1-GFP. Cells were cocultured with the indicated "stromal cells" in the presence or absence of doxycycline (Dox) and siRNA targeting ICMT or a nontargeting control. The stromal cells were NIH3T3 (3Т3) with or without expression of the Notch1 ligand Jagged-2 (J2). The $\gamma$-secretase inhibitor compound E was included as a control and completely blocked Notch signaling. All ratios were normalized to the maximum in each experiment. Data shown are mean \pm SEM, $n=3$. Panel on right shows a representative knockdown by immunoblotting for ICMT and a control (ERK1/2). (B) Wings of $D$. melanogaster transgenic for UAS-shSte14, a GAL4-responsive hairpin that silences Icmt. GAL4 expression was driven by a wing-specific (Ap-GAL4) promoter. ICMT deficiency in the developing wing phenocopies the terminal vein bifurcation (arrows) and thickened cross-vein (arrowhead) observed in the wings of Delta $(D /)$ flies deficient for the Notch ligand. (C) GAL4 expression driven with an eye-specific (GMR-GAL4) promoter. The rough eye phenotype is also consistent with that seen in Notch loss-of-function alleles, including DI. (D) Wing imaginal discs from third instar larvae expressing shSte14 in heat shock-induced clones marked with GFP and stained for CUT, a Notch-dependent gene product. Where the GFP-positive clone intersects the line of CUT staining there is a decrease in the number of CUT-positive cells (enlargement). Scale bar: $50 \mu \mathrm{m}$. 
prenylation of RAS (8) rather than prenylation-independent RAS oncogenicity. Thus, strategies that effectively curtail CaaX processing are still considered among the most viable approaches to anti-RAS therapeutics. Accordingly, ICMT is considered a good drug target, and enthusiasm for this approach has been bolstered by several preclinical studies that have found a requirement for ICMT in KRAS-mediated cellular transformation (9) and oncogenesis in vivo (10) as well as in studies suggesting that pharmacologic inhibition of ICMT blocks RAS-dependent transformation $(44,45)$. We set out to solidify this view by studying the role of ICMT in a mouse model of PDA that more faithfully recapitulates human pathology than the cancer models previously examined. To our great surprise, we discovered that ICMT deficiency accelerates the development of both PanINs and PDA in the Pdx1-Cre; LSL-Kras ${ }^{G 12 D}$ model.

In explaining the consequences of ICMT deficiency, one must consider that RAS is not the only substrate of the enzyme. Indeed, there are on the order of $300 \mathrm{CaaX}$ proteins and another $19 \mathrm{RAB}$ proteins terminating in a CXC sequence that are putative substrates of ICMT (17-19). Many of the CaaX substrates are signaling molecules, and $\mathrm{RAB}$ proteins are required for vectorial vesicular transport and biogenesis of membrane-bound organelles (46). Our observation that all pancreatic structures develop normally in animals in which ICMT is deleted in pancreatic progenitor cells at E8.5 is therefore somewhat surprising and suggests that, despite the conservation of ICMT throughout eukaryotic evolution, a large subset of ICMT substrates retain biological activity in the absence of carboxyl methylation. Pancreatic development in the absence of ICMT is concordant with the development of many other tissues, including a beating heart, in Icmt-null embryos, which die in utero at E10.5 $(47,48)$.

There are several possible causes for the acceleration of the neoplastic lesions in Pdx1-Cre;LSL-Kras ${ }^{G 12 D}$ mice as a consequence of ICMT deficiency. One possibility is that, in the pancreas, unmethylated oncogenic KRAS could be hyperactive as a consequence of altered subcellular distribution. Indeed, we have shown that RAS proteins are able to signal from endomembranes (49). However, ICMT deficiency causes an increase in cytosolic KRAS rather than mislocalization to endomembranes $(29,50)$, and cytosolic RAS is unable to signal (5). On the contrary, cytosolic, oncogenic RAS has been shown to have a dominant-negative effect on the MAPK pathway (51). The most compelling evidence against unmethylated KRAS gaining function derives from our cell-based studies, which showed that KRAS is unable to transform rodent fibroblasts without ICMT (9) and that there is an ameliorating effect of ICMT deficiency on KRAS-driven myeloproliferative disease and lung tumors in mice (10).

Another possibility is that the acceleration of PanINs in the absence of ICMT might be due to an enhanced stromal reaction. PDA is characterized by an intense desmoplastic reaction that includes inflammation and fibrosis (26). Moreover, pancreatitis is a well-established risk factor for pancreatic cancer (32). Importantly, cerulein-induced acute pancreatitis is associated with ADM and markedly accelerates KRAS ${ }^{\text {G12D-induced PanIN }}$ progression in a manner very similar to that of ICMT deficiency (52). We observed an exacerbation of both inflammatory cell infiltrates and fibrosis in ICMT-deficient pancreata expressing KRAS ${ }^{\mathrm{G} 12 \mathrm{D}}$. Thus, it is possible that ICMT-deficient acinar and/or ductal cells either support intensified inflammation or are slower to resolve inflammation and that this is the trigger for accelerated PanINs in Icmt $t^{f l / f l x} ; P d x 1-C r e ; L S L-K r a s^{G 12 D}$ mice. However, we observed no evidence of increased inflammation in ICMT-deficient pancreata in the absence of oncogenic Kras, even when the mice were allowed to age for a year or more. Moreover, we observed no effect of ICMT deficiency either on the severity of inflammation or on the rate at which the inflammation resolved in cerulein-induced acute or chronic pancreatitis. Although these observations do not rule out an effect of ICMT deficiency on the stromal reaction of PanINs in Pdx1-Cre;LSLKras $^{G 12 D}$ mice, they argue against ICMT deficiency promoting pancreatic inflammation.

A third possibility is that the acceleration of pancreatic neoplasia in Icmt $t^{f l} / f l x ; P d x 1-C r e ; L S L-K r a s^{G 12 D}$ mice could be a consequence of effects on ICMT substrates other than KRAS. That is, among the many substrates of ICMT that are signaling molecules, one or more may function as a tumor suppressor in the pancreas and may require carboxyl methylation for biological activity. In this model, ICMT could be considered a tumor suppressor, but one that works indirectly by methylating molecules that are themselves tumor suppressors. Differential expression of substrates might explain why ICMT could have tumor suppressor-like function in one tissue (e.g., pancreas) and not another (e.g., bone marrow). Like many tumor suppressors, loss of function of the relevant ICMT substrate would not be sufficient to provoke neoplasia but, when combined with the activation of an oncogene such as Kras ${ }^{G 12 D}$, tumors would be initiated or accelerated. This would explain why no PanINs were observed in the pancreata of Icm $t^{f l x / f l x} ; P d x 1$-Cre mice. The fact that a dominant-negative mutation of p53 did not accelerate PDA to a significant degree when combined with ICMT deficiency in the Pdx1-Cre;LSL-Kras ${ }^{G 12 D}$ model does not argue against the tumor suppressor-like activity of ICMT, since the inactivation of $\mathrm{p} 16^{\text {INK4A }}$ also did not accelerate the tumors seen in this model when combined with p53 inactivation (53). Our observation that ICMT-deficient PDECs have a slight growth advantage over wild-type PDECs, even in the absence of oncogenic Ras, and that like KRAS-transformed PDECs, are protected from premature senescence, provides compelling evidence for a cell-autonomous effect of ICMT deficiency in PDECs and is consistent with the notion of a tumor suppressor-like activity of ICMT. The suppression of $\mathrm{p} 16^{\mathrm{INK} 4 \mathrm{~A}}$ expression in ICMT-deficient cells is also consistent with a requirement for ICMT in pathways that control the cell cycle, a hallmark of tumor suppressors (54).

There is ample precedence for signaling molecules that can behave as either oncogenes or tumor suppressors, depending on the cellular context. Notch proteins are examples of such molecules. NOTCH1 is a well-established oncogene in T cell acute lymphoblastic leukemia, but acts as a tumor suppressor in skin (42). The complexity of the opposing effects of Notch 1 in different tissues also extends to different Notch isoforms in the same tissue: whereas Notch2 is required for PanIN progression in Pdx1-Cre;LSL-Kras ${ }^{G 12 D}$ mice, Notch1 is not (55), and in fact, the latter isoform behaves like a tumor suppressor in this model (15). The similarities between the effects of Notch 1 and ICMT deficiency in the Pdx1-Cre;LSL-Kras ${ }^{G 12 D}$ mouse model were striking and included not only accelerated PanINs but also papillomas below the pinna on the faces of these animals. This observation strongly suggested that ICMT and Notch1 deficiency affect a common pathway, and the simplest explanation is to hypothesize that Notch 1 signaling requires ICMT activity. We confirmed 
this hypothesis both cell biologically in human osteosarcoma cells and genetically in Drosophila wing development. As currently understood, there is no obvious ICMT substrate in the Notch1 signaling pathway, although it is unlikely that the full details of Notch signaling have been elucidated (42). There is some evidence that endocytosis is required for Notch signaling (56). If so, one could argue that endocytosis requires small GTPases of the RAB and RHO families that are substrates of ICMT. Because of the large number of candidates, the identification of ICMT substrates that play a role in Notch signaling will require extensive investigation but will likely reveal important new features of this highly conserved pathway.

Although at first glance, targeting the three enzymes that modify CaaX sequences, including ICMT, appears to be a straightforward approach to inhibit RAS function, our results reveal a complexity that obfuscates rational drug design. The complexity likely arises from the large repertoire of signaling molecules that are modified by the CaaX processing enzymes. We believe that our results implicate for the first time the Notch pathway as one that must be considered when attempting to predict the pleiotropic effects of ICMT inhibition. Given the fact that in some models, ICMT deficiency ameliorates tumors (10) and that ICMT inhibitors have been shown to have antitumor activity $(44,45)$, does our surprising observation that ICMT deficiency exacerbates KRAS-driven PanINs in one mouse model mean that ICMT inhibitors cannot be developed as drugs? This will depend on how generalized the results are that we report here.

\section{Methods}

Mouse breeding. Mice with a conditional Icmt allele (Icmt $\left.t^{f x}\right)$ (9) were bred with mice harboring a latent $C r e$-inducible oncogenic $L S L-K r a s^{G 12 D}$ allele from the endogenous promoter (57) to generate Icmt ${ }^{f l x / f l x} ; L S L-K r a s^{G 12 D}$ mice. These mice were then bred with mice containing a Pdx1-Cre transgene (11) (MMHCC; National Cancer Institute [NCI], Frederick, Maryland, USA) that induces the expression of Cre recombinase in pancreatic progenitor cells. These crosses generated experimental Icmt $t^{f l x f l x}$; LSL-Kras ${ }^{G 12 D} ; P d x 1$-Cre mice and control Icm $t^{\ell x /+} ; L S L-K r a s^{G 12 D} ; P d x 1$-Cre mice that retained a wild-type copy of $I c m t$. In a separate cross, mice containing Icmt $t^{\ell x}, L S L-K r a s^{G 12 D}$, and Pdx1-Cre alleles were bred with mice containing a Cre-inducible dominant-negative $L S L-p 53^{R 172 H}$ allele (58) (MMHCC; NCI) to generate experimental Icm t $t^{f x / f l x} ; L S L-K r a s^{G 12 D} ; P d x 1$-Cre; $L S L-p 53^{R 172 H}$ and control Icm t flx/flx; $; S L-K r a s^{G 12 D} ; P d x 1-C r e ; L S L-p 53^{R 172 H}$ mice. In a third cross, mice containing a Rosa26-LSL-LacZ reporter allele (21) (JAX mice) were bred with $I c m t^{f l x} / f x ; P d x 1$-Cre mice. The gene nomenclature used for the different groups of mice refers to the genotype detected in tail DNA at weaning. All mice were maintained on a $129 / \mathrm{Sv}$ and C57BL/6 mixed genetic background. Intraperitoneal glucose tolerance tests in mice were carried out as described (59). All animal care and procedures followed NIH guidelines and were approved by the IACUC of New York University School of Medicine.

Genotyping. Genotyping was performed by PCR amplification of genomic DNA extracted from mouse tails, tumors, tissues and cultured cells. The Icmt $t^{\text {tx }}$ allele was detected with forward primer 5'-GGGCGGGACGGACAG-3' and reverse primer 5'-ATGCGCATCTGCCTAAGCTG-3', which yielded a 600-bp fragment from the Icm thx allele and a 538-bp fragment from the Icmt $t^{+}$allele. The Icmt $t^{+}$allele was detected with forward primer 5'-CCGCTCGCTCCGAATCCAG-3' and reverse primer 5'-CTCGTAAACCGACCACCAAT-3', which yielded a 600-bp fragment. The stop cassette in the $L S L-K$ ras $^{G 12 D}$ allele was detected with forward primer 5'-CCTTTACAAGCGCACGCAGACTGTAGA-3' and reverse primer 5'-AGCTAGCCACCATGGCTTGAGTAAGTCTGCA-3', yielding a 600-bp amplified fragment. The activated $\operatorname{Kras}^{G 12 D}$ allele was detected with forward primer $5^{\prime}$-GGGTAGGTGTTGGGATAGCTG-3' and reverse primer 5'-TCCGAATTCAGTGACTACAGATGTACAGAG-3', yielding a 320 -bp fragment from the activated $K_{r a s}{ }^{G 12 D}$ allele and a 280-bp fragment from the wild-type Kras allele. The $L S L-p 53^{R 172 H}$ allele and wild-type $p 53$ allele were detected with primers 5 '-AGCTAG CCACCATGGCTTGAGTAAGTCTGCA-3', 5'-CTTGGAGACATAGCCACACTG-3', and 5'-TTACACATCCAGCCTCTGTGG-3', which generated a 270 -bp product for the $L S L-p 53^{R 172 H}$ allele and a 166-bp product for the wild-type allele. The Pdx1-Cre transgene was detected with forward primer 5 '-CTGGACTACATCTTGAGTTGC- $3^{\prime}$ and reverse primer $5^{\prime}$-GGTGTACGGTCAGTAAATTTG-3', yielding a 650-bp amplified product. The Rosa26-LSLLacZ allele and corresponding wild-type allele were detected with primers 5'-GCGAAGAGTTTGTCCTCAACC-3', 5'-AAAGTCGCTCTGAGTTGTTAT-3', and 5'-GGAGCGGGAGAAATGGATATG-3', which generated a 340-bp product for the Rosa26-LSL-LacZ allele and a 650-bp product for the wild-type allele.

In vivo experiments. The LSL-Kras ${ }^{G 12 D} ; P d x 1$-Cre and LSL-Kras ${ }^{G 12 D} ; P d x 1$ Cre; $L S L-p 53^{R 172 H}$ mouse models of pancreatic cancer have been described previously $(11,24)$. To measure PanIN progression over time, all pancreatic ducts were scored as normal, PanIN-1A, PanIN-1B, PanIN-2, or PanIN-3 in representative H\&E-stained sections from mice of varying ages according to established criteria (23). Only the highest-grade lesion in each pancreatic lobule was evaluated. Sections were also examined for evidence of PDA according to established criteria (60). To induce acute or chronic pancreatitis, mice were given 7 hourly i.p. injections of $50 \mu \mathrm{g} / \mathrm{kg}$ cerulein (Sigma-Aldrich) for either 2 days (acute) or thrice weekly for 3 weeks (chronic). Mice were sacrificed either 5 hours or 3 to 7 days after the final injection.

PDEC isolation and culture. PDECs were isolated from 2- to 3-monthold mice of the genotypes Icm t $t^{f x /+}, \operatorname{Icm} t^{f l x / f l x}, \operatorname{Icm} t^{f x /+} ; L S L-K r a s^{G 12 D}$, and $I c m t^{f l x /+} ; L S L-K r a s^{G 12 D}$ and cultured and embedded in 3D Matrigel (BD), as described previously (36). Cells were infected with adenovirus expressing Cre (Ad5CMVCre; University of Iowa, Gene Transfer Vector Core, Iowa City, Iowa, USA) to induce recombination of the floxed alleles as described (37). For all experiments, the PDECs were transferred to $1 \%$ Matrigel-coated tissue $2 \mathrm{D}$ culture plates and allowed to proliferate. To measure the proliferation rate of PDECs, the cells were trypsinized after 3 days in 2D culture, and 5,000 cells were transferred to 96-well plates. Cell numbers were measured with an MTS assay (Promega). After 12 days in 2D culture, cells were stained for the expression of senescence-associated $\beta$-gal as described (37) and counterstained with $1 \mu \mathrm{g} / \mathrm{ml}$ Hoechst 33342 (Invitrogen). Differential interference contrast (DIC) and fluorescent images of cells were taken with a Zeiss Axiovert 200 microscope using a $\times 20$ objective. The area of blue staining per field was measured using MetaMorph software (Molecular Devices) and normalized to the number of nuclei in each field.

Pancreas fractionation. Pancreatic tissue was homogenized by Dounce in hypotonic buffer (10 mM HEPES, pH 7.3, $100 \mathrm{mM} \mathrm{KCl,} 3 \mathrm{mM} \mathrm{NaCl}, 35$ $\mathrm{mM} \mathrm{MgCl}_{2}$ ). Membrane (P100) and cytosolic fractions (S100) were isolated from postnuclear supernatants by ultracentrifugation at $100,000 \mathrm{~g}$ for 30 minutes. An in vitro assay for ICMT activity was carried out as described (22) using $10 \mu \mathrm{g}$ of pancreas membrane protein. P100 and S100 fractions $(100 \mu \mathrm{g})$ were also loaded onto SDS-PAGE gels for subsequent immunoblot analysis.

Immunoblot. Cells were lysed in Tris-Glycine SDS Sample Buffer (Invitrogen), and the lysates were separated by electrophoresis in SDS polyacrylamide gels, then transferred to nitrocellulose membranes. After blocking, the membranes were incubated with the following primary antibodies: rabbit anti-KRAS, rabbit anti-p16 ${ }^{\mathrm{INK} 4 \mathrm{~A}}$, rabbit anti-ERK1, goat 
anti-Na $\mathrm{K}^{+}$-ATPase, rabbit anti-RhoGDI, goat anti- $\beta$-1,4-gal-T4 (all from Santa Cruz Biotechnology Inc.), mouse anti- $\beta$-tubulin (E7, developed by M. Klymkowsky and obtained from Developmental Studies Hybridoma Bank), rabbit anti-Notch1 (Cell Signaling Technology), and mouse antiRAS (RAS10; Calbiochem). Secondary antibodies were IRDye 800-conjugated goat anti-rabbit or IRDye 680 -conjugated goat anti-mouse (LiCOR). Blots were visualized with the Odyssey Infrared Imaging System (Li-COR) and quantified using Odyssey software.

GST-RBD pulldown for active RAS. HeLa cells were serum starved in $0.5 \%$ serum overnight before stimulation with $10 \mathrm{ng} / \mathrm{ml} \mathrm{EGF}$ for 3 minutes. Cells were lysed in magnesium lysis buffer (25 mM HEPES, pH 7.5, 125 $\mathrm{mM} \mathrm{NaCl}, 10 \mathrm{mM} \mathrm{MgCl}$, $1 \mathrm{mM}$ EDTA, 1\% IGEPAL CA-630, and 10\% glycerol). GST-RBD pulldown of active GTP-bound RAS was carried out as described $(61,62)$. The lysed cellular material $(1 \%)$ was saved prior to pulldown as a loading control for the total amount of RAS present.

Histology. Mouse tissues were fixed in $10 \%$ formalin overnight. After fixation, tissues were dehydrated in 50\% to $100 \%$ ethanol, cleared in xylene, and embedded in paraffin. Sections $(5-\mu \mathrm{m})$ were stained with either $\mathrm{H} \& \mathrm{E}$ or Masson's trichrome and visualized by light microscopy using a Zeiss Axiovert 200 microscope. Immunohistochemistry on paraffin sections was performed as described (37) using the following antibodies: mouse anti- $\beta$-catenin (BD), rabbit anti-pERK, anti- $\alpha$-smooth muscle actin (Novus Biologicals), and anti-Ki67 (Cell Signaling Technology). For immunofluorescence, samples of pancreas were embedded in OCT compound (Tissue-Tek) and snap-frozen. Sections $(8-\mu \mathrm{m})$ were fixed in $4 \%$ paraformaldehyde for 10 minutes, permeabilized in $0.2 \%$ Triton X-100 for 20 minutes, and blocked with $1 \%$ IgG-free BSA and $50 \mu \mathrm{g} / \mathrm{ml}$ donkey IgG (Jackson ImmunoResearch) for 1 hour. The sections were incubated with primary antibodies diluted in $1 \%$ BSA and $0.1 \%$ Tween20 overnight at $4{ }^{\circ} \mathrm{C}$, and the Alexa Fluor-labeled secondary antibodies (Invitrogen) were diluted in 1\% BSA for 1 hour. The sections were washed in PBS containing $1 \mu \mathrm{g} / \mathrm{ml}$ Hoechst 33342 (Invitrogen) and mounted with fluorescent mounting media (DakoCytomation). Drosophila wing discs were fixed and processed in the same way before mounting with Vectashield (Vector Laboratories). Tissues were visualized by fluorescence microscopy using a Zeiss Axiovert 200 microscope or a Zeiss LSM 510 confocal microscope. The following antibodies were used: rat anti-CD45 (BD); rat anti-CK19 (Troma-III, developed by Rolf Kemler and obtained from Developmental Studies Hybridoma Bank); goat anti-amylase (Santa Cruz Biotechnology Inc.); and mouse anti-CUT (2B10, developed by Rubin, G.M. and obtained from Developmental Studies Hybridoma Bank). Staining for $\beta$-gal expression was carried out on 8 - $\mu \mathrm{m}$ frozen sections of pancreatic tissue. Sections were fixed in $2 \%$ formaldehyde $/ 0.2 \%$ glutaraldehyde in PBS for 5 minutes, washed in PBS, and stained at $37^{\circ} \mathrm{C}$ for 12 to 16 hours in X-gal solution $(1 \mathrm{mg} / \mathrm{ml} \mathrm{X-Gal,} 5 \mathrm{mM}$ potassium ferrocyanide, $5 \mathrm{mM}$ potassium ferricyanide, and $1 \mathrm{mM} \mathrm{MgCl}_{2}$ in $\mathrm{PBS}$ ). Slides were counterstained with eosin, mounted with Permount (Fisher), and imaged with a wide-field fluorescence microscope.
Luciferase assays. Luciferase assays for Notch 1 activity were carried out as described (63). All assays were performed in 96-well plates, and luciferase expression was measured using a Dual-Glo Luciferase kit (Promega) and detected in a plate reader. Icmt knockdown was performed with an siRNA SMARTpool (Dharmacon). A nontargeting siRNA pool was used as a negative control. Knockdown of ICMT was validated in each cell line tested by immunoblot using an ICMT antibody (Proteintech). Compound E (CE) was obtained from Enzo Lifesciences.

Drosophila stocks and genetics. The UAS-ShSte14 stock was obtained from The Bloomington Drosophila Stock Center (stock number 34996). Ap-GAL4, MS1096-GAL4, and GMR-GAL4 lines were obtained from Ramanuj DasGupta (NYU School of Medicine). UAS-shSte14 males were crossed with virgin females from the GAL4 driver lines at $29^{\circ} \mathrm{C}$. For heatshock experiments, $y w$; Act $>y^{+}>$Gal4, UAS-GFP males were crossed with $y w b s-$ flp122; UAS-ShSte 14 virgins at $25^{\circ} \mathrm{C}$. Larvae were heat shocked at $37^{\circ} \mathrm{C}$ for 10 minutes approximately 30 hours after egg-laying. Wing imaginal discs were dissected from third instar larvae.

Statistics. PanIN frequencies were compared by means of a generalized linear model for binomial data using age and genotype as covariates. All analyses were carried out using $R$ (64). All other statistical analyses were carried out using a Student's $t$ test. $P$ values of less than 0.05 were considered significant.

\section{Acknowledgments}

We thank Stephen Blacklow for providing expert advice as well as cell lines and reagents for the Notch1 luciferase assay. We thank Jonathan Court for statistical analysis of data. We thank Cynthia Loomis, Luis Chiriboga, and the NYU Langone Medical Center Histopathology and Immunohistochemistry Core Facilities for their assistance with specimen preparation and histology. We thank Jessica Treisman and Ramanuj DasGupta for fly stocks and discussions. We thank Rebecca Zuckerman and Christopher Graffeo for technical assistance. This work was supported by NIH Grants CA118495, CA116034, and GM055279 and by funding from the Jeffrey Rosenzweig Foundation for Pancreatic Cancer Research (to M.R. Philips). Support was also provided by NIH Awards DK085278 and CA155649 (to G. Miller) and 5R01CA55360 and 5R01GM078266 (to D. Bar-Sagi). This work was also supported in part by NCI grant 5P30CA016087-32.

Received for publication January 16, 2013, and accepted in revised form August 8, 2013.

Address correspondence to: Mark Philips, New York University School of Medicine, NYU Cancer Institute, Smilow 1207, 522 First Ave., New York, New York, USA. Phone: 212.263.7404; Fax: 212.263.9210; E-mail: philim01@med.nyu.edu.
1. Bos JL. ras oncogenes in human cancer: a review. Cancer Res. 1989;49(17):4682-4689.

2. Downward J. Targeting RAS signalling pathways in cancer therapy. Nat Rev Cancer. 2003;3(1):11-22.

3. Vigil D, Cherfils J, Rossman KL, Der CJ. Ras superfamily GEFs and GAPs: validated and tractable targets for cancer therapy? Nat Rev Cancer. 2010; 10(12):842-857.

4. Barbacid M. ras genes. Annu Rev Biochem. 1987; 56:779-827.

5. Willumsen BM, Christensen A, Hubbert NL, Papageorge AG, Lowy DR. The $\mathrm{p} 21$ ras C-terminus is required for transformation and membrane association. Nature. 1984;310(5978):583-586.
6. Wright LP, Philips MR. Thematic review series: lipid posttranslational modifications. CAAX modification and membrane targeting of Ras. J Lipid Res. 2006;47(5):883-891.

7. Cox AD, Der CJ. Farnesyltransferase inhibitors: promises and realities. Curr Opin Pharmacol. 2002; 2(4):388-393.

8. Whyte DB, et al. K- and N-Ras are geranylgeranylated in cells treated with farnesyl protein transferase inhibitors. J Biol Chem. 1997;272(22):14459-14464.

9. Bergo MO, et al. Inactivation of Icmt inhibits transformation by oncogenic K-Ras and B-Raf. J Clin Invest. 2004;113(4):539-550.

10. Wahlstrom AM, et al. Inactivating Icmt ameliorates
K-RAS-induced myeloproliferative disease. Blood. 2008;112(4):1357-1365.

11. Hingorani SR, et al. Preinvasive and invasive ductal pancreatic cancer and its early detection in the mouse. Cancer Cell. 2003;4(6):437-450.

12. Yonezawa S, Higashi M, Yamada N, Goto M. Precursor lesions of pancreatic cancer. Gut Liver. 2008;2(3):137-154.

13. Almoguera C, Shibata D, Forrester K, Martin J, Arnheim N, Perucho M. Most human carcinomas of the exocrine pancreas contain mutant c-K-ras genes. Cell. 1988;53(4):549-554.

14. Shi $\mathrm{C}$, et al. KRAS2 mutations in human pancreatic acinar-ductal metaplastic lesions are limited 
to those with PanIN: implications for the human pancreatic cancer cell of origin. Mol Cancer Res. 2009; 7(2):230-236.

15. Hanlon L, et al. Notch1 functions as a tumor suppressor in a model of K-ras-induced pancreatic ductal adenocarcinoma. Cancer Res. 2010;70(11):4280-4286.

16. Mazur PK, et al. Identification of epidermal Pdx1 expression discloses different roles of Notch 1 and Notch2 in murine Kras(G12D)-induced skin carcinogenesis in vivo. PLoS One. 2010;5(10):e13578.

17. Maurer-Stroh S, Koranda M, Benetka W, Schneider G, Sirota FL, Eisenhaber F. Towards complete sets of farnesylated and geranylgeranylated proteins. PLoS Comput Biol. 2007;3(4):e66.

18. Ashar HR, et al. Farnesyl transferase inhibitors block the farnesylation of CENP-E and CENP-F and alter the association of CENP-E with the microtubules. J Biol Chem. 2000;275(39):30451-30457.

19. Reid TS, Terry KL, Casey PJ, Beese LS. Crystallographic analysis of CaaX prenyltransferases complexed with substrates defines rules of protein substrate selectivity. J Mol Biol. 2004;343(2):417-433.

20. Gannon M, Herrera PL, Wright CV. Mosaic Cremediated recombination in pancreas using the $\mathrm{pdx}-1$ enhancer/promoter. Genesis. 2000;26(2):143-144

21. Soriano P. Generalized lacZ expression with the ROSA26 Cre reporter strain. Nat Genet. 1999; 21(1):70-71.

22. Choy E, Philips M. Expression and activity of human prenylcysteine-directed carboxyl methyltransferase. Methods Enzymol. 2000;325:101-114.

23. Hruban RH, et al. An illustrated consensus on the classification of pancreatic intraepithelial neoplasia and intraductal papillary mucinous neoplasms. Am J Surg Pathol. 2004;28(8):977-987.

24. Hingorani SR, et al. Trp53R172H and KrasG12D cooperate to promote chromosomal instability and widely metastatic pancreatic ductal adenocarcinoma in mice. Cancer Cell. 2005;7(5):469-483.

25. Zhu L, Shi G, Schmidt CM, Hruban RH, Konieczny SF. Acinar cells contribute to the molecular heterogeneity of pancreatic intraepithelial neoplasia. Am J Pathol. 2007;171(1):263-273.

26. Chu GC, Kimmelman AC, Hezel AF, DePinho RA. Stromal biology of pancreatic cancer. J Cell Biochem. 2007;101(4):887-907.

27. Bachem MG, et al. Pancreatic carcinoma cells induce fibrosis by stimulating proliferation and matrix synthesis of stellate cells. Gastroenterology. 2005;128(4):907-921.

28. Michaelson D, et al. Postprenylation CAAX processing is required for proper localization of Ras but not Rho GTPases. Mol Biol Cell. 2005; 16(4):1606-1616.

29. Bergo MO, Leung GK, Ambroziak P, Otto JC, Casey PJ, Young SG. Targeted inactivation of the isoprenylcysteine carboxyl methyltransferase gene causes mislocalization of K-Ras in mammalian cells. J Biol Chem. 2000;275(23):17605-17610.

30. Ijichi $\mathrm{H}$, et al. Aggressive pancreatic ductal adenocarcinoma in mice caused by pancreas-specific blockade of transforming growth factor-beta signaling in cooperation with active Kras expression.
Genes Dev. 2006;20(22):3147-3160.

31. Guerra C, et al. Chronic pancreatitis is essential for induction of pancreatic ductal adenocarcinoma by K-Ras oncogenes in adult mice. Cancer Cell. 2007;11(3):291-302.

32. Lowenfels AB, Maisonneuve P, Lankisch PG. Chronic pancreatitis and other risk factors for pancreatic cancer. Gastroenterol Clin North Am. 1999;28(3):673-685, x.

33. Lampel M, Kern HF. Acute interstitial pancreatitis in the rat induced by excessive doses of a pancreatic secretagogue. Virchows Arch A Pathol Anat Histol. 1977;373(2):97-117.

34. Watanabe O, Baccino FM, Steer ML, Meldolesi J. Supramaximal caerulein stimulation and ultrastructure of rat pancreatic acinar cell: early morphological changes during development of experimental pancreatitis. Am J Physiol. 1984; 246(4 pt 1):G457-467.

35. Bedrosian AS, et al. Dendritic cells promote pancreatic viability in mice with acute pancreatitis. Gastroenterology. 2011;141(5):1915-1914.

36. Agbunag C, Lee KE, Buontempo S, Bar-Sagi D. Pancreatic duct epithelial cell isolation and cultivation in two-dimensional and three-dimensional culture systems. Methods Enzymol. 2006;407:703-710.

37. Lee KE, Bar-Sagi D. Oncogenic KRas suppresses inflammation-associated senescence of pancreatic ductal cells. Cancer Cell. 2010;18(5):448-458.

38. Nicolas M, et al. Notch1 functions as a tumor suppressor in mouse skin. Nat Genet. 2003;33(3):416-421.

39. Demehri S, Turkoz A, Kopan R. Epidermal Notch1 loss promotes skin tumorigenesis by impacting the stromal microenvironment. Cancer Cell. 2009;16(1):55-66

40. Parody TR, Muskavitch MA. The pleiotropic function of Delta during postembryonic development of Drosophila melanogaster. Genetics. 1993;135(2):527-539.

41. Micchelli CA, Rulifson EJ, Blair SS. The function and regulation of cut expression on the wing margin of Drosophila: Notch, Wingless and a dominant negative role for Delta and Serrate. Development. 1997;124(8):1485-1495.

42. Radtke F, Raj K. The role of Notch in tumorigenesis: oncogene or tumour suppressor? Nat Rev Cancer. 2003;3(10):756-767.

43. Ahearn IM, Haigis K, Bar-Sagi D, Philips MR. Regulating the regulator: post-translational modification of RAS. Nat Rev Mol Cell Biol. 2012;13(1):39-51.

44. Winter-Vann AM, et al. A small-molecule inhibitor of isoprenylcysteine carboxyl methyltransferase with antitumor activity in cancer cells. Proc Nat Acad Sci U S A. 2005;102(12):4336-4341.

45. Judd WR, et al. Discovery and SAR of methylated tetrahydropyranyl derivatives as inhibitors of isoprenylcysteine carboxyl methyltransferase (ICMT). JMed Chem. 2011;54(14):5031-5047.

46. Grosshans BL, Ortiz D, Novick P. Rabs and their effectors: achieving specificity in membrane traffic. Proc Natl Acad Sci US A. 2006;103(32):11821-11827.

47. Bergo MO, et al. Isoprenylcysteine carboxyl methyltransferase deficiency in mice. J Biol Chem.
2001;276(8):5841-5845

48. Lin X, Jung J, Kang D, Xu B, Zaret KS, Zoghbi H. Prenylcysteine carboxylmethyltransferase is essential for the earliest stages of liver development in mice. Gastroenterology. 2002;123(1):345-351.

49. Chiu VK, et al. Ras signalling on the endoplasmic reticulum and the Golgi. Nat Cell Biol. 2002;4(5):343-350.

50. Michaelson D, et al. Postprenylation CAAX processing is required for proper localization of Ras but not Rho GTPases. Mol Biol Cell. 2005; 16(4):1606-1616

51. Fiordalisi JJ, Holly SP, Johnson RL, Parise LV, Cox AD. A distinct class of dominant negative Ras mutants: cytosolic GTP-bound Ras effector domain mutants that inhibit Ras signaling and transformation and enhance cell adhesion. J Biol Chem. 2002;277(13):10813-10823.

52. Carriere C, Young AL, Gunn JR, Longnecker DS, Korc M. Acute pancreatitis markedly accelerates pancreatic cancer progression in mice expressing oncogenic Kras. Biochem Biophys Res Commun. 2009;382(3):561-565.

53. Bardeesy $\mathrm{N}$, et al. Both p16(Ink4a) and the p19(Arf)-p53 pathway constrain progression of pancreatic adenocarcinoma in the mouse. Proc Natl Acad Sci U S A. 2006;103(15):5947-5952.

54. Sherr CJ. Principles of tumor suppression. Cell. 2004;116(2):235-246

55. Mazur PK, et al. Notch2 is required for progression of pancreatic intraepithelial neoplasia and development of pancreatic ductal adenocarcinoma. Proc Natl Acad Sci U S A. 2010;107(30):13438-13443.

56. Le Borgne R, Bardin A, Schweisguth F. The roles of receptor and ligand endocytosis in regulating Notch signaling. Development. 2005;132(8):1751-1762.

57. Jackson EL, et al. Analysis of lung tumor initiation and progression using conditional expression of oncogenic K-ras. Genes Dev. 2001; 15(24):3243-3248.

58. Olive KP, et al. Mutant p53 gain of function in two mouse models of Li-Fraumeni syndrome. Cell. 2004;119(6):847-860

59. Heikkinen S, Argmann CA, Champy MF, Auwerx J. Evaluation of glucose homeostasis. Curr Protoc Mol Biol. 2007; Chapter 29:Unit 29B.3.

60. Hruban RH, Fukushima N. Pancreatic adenocarcinoma: update on the surgical pathology of carcinomas of ductal origin and PanINs. Mod Pathol. 2007;20(suppl 1):S61-S70.

61. Ahearn IM, et al. FKBP12 binds to acylated $\mathrm{H}$-ras and promotes depalmitoylation. Mol Cell. 2011;41(2):173-185.

62. de Rooij J, Bos JL. Minimal Ras-binding domain of Raf1 can be used as an activation-specific probe for Ras. Oncogene. 1997;14(5):623-625.

63. Aste-Amezaga M, et al. Characterization of Notch1 antibodies that inhibit signaling of both normal and mutated Notch 1 receptors. PLoS One. 2010;5(2):e9094.

64. R Development Core Team. R: A Language Environment For Statistical Computing. Vienna, Austria: R Foundation for Statistical Computing; 2012. 